Portland State University

PDXScholar

$12-2018$

\title{
Vehicle Sensor Data (VSD) Based Traffic Control in Connected Automated Vehicle (CAV) Environment
}

Xianfeng Terry Yang

University of Utah

Zhao Zhang

University of Utah

Zhehao Zhang

University of Utah

Follow this and additional works at: https://pdxscholar.library.pdx.edu/trec_reports

Part of the Transportation Commons, Urban Studies Commons, and the Urban Studies and Planning Commons

Let us know how access to this document benefits you.

\section{Recommended Citation}

Yang, Xianfeng, Zhang, Zhehao and Zhang, Zhao. Vehicle Sensor Data (VSD) Based Traffi Control in Connected Automated Vehicle (CAV) Environment. NITC-SS-1175. Portland, OR: Transportation Research and Education Center (TREC), 2018. https://doi.org/10.15760/trec.212

This Report is brought to you for free and open access. It has been accepted for inclusion in TREC Final Reports by an authorized administrator of PDXScholar. Please contact us if we can make this document more accessible: pdxscholar@pdx.edu. 

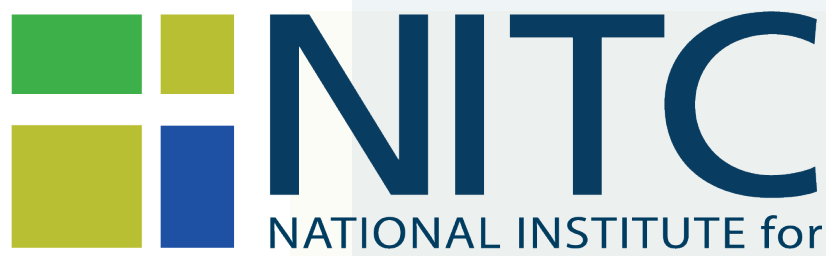

NATIONAL INSTITUTE for

TRANSPORTATION and COMMUNITIES

FINAL REPORT

Vehicle Sensor Data (VSD)-Based Traffic Control in Connected Automated Vehicle (CAV) Environment

NITC-SS-1175 December 2018

NITC is a U.S. Department of Transportation national university transportation center.

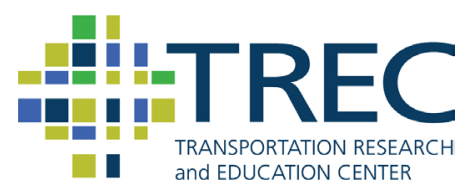




\title{
VEHICLE SENSOR DATA (VSD)-BASED TRAFFIC CONTROL IN CONNECTED AUTOMATED VEHICLE (CAV) ENVIRONMENT
}

\author{
Final Report \\ NITC-SS-1175 \\ by \\ Xianfeng Terry Yang \\ University of Utah
}

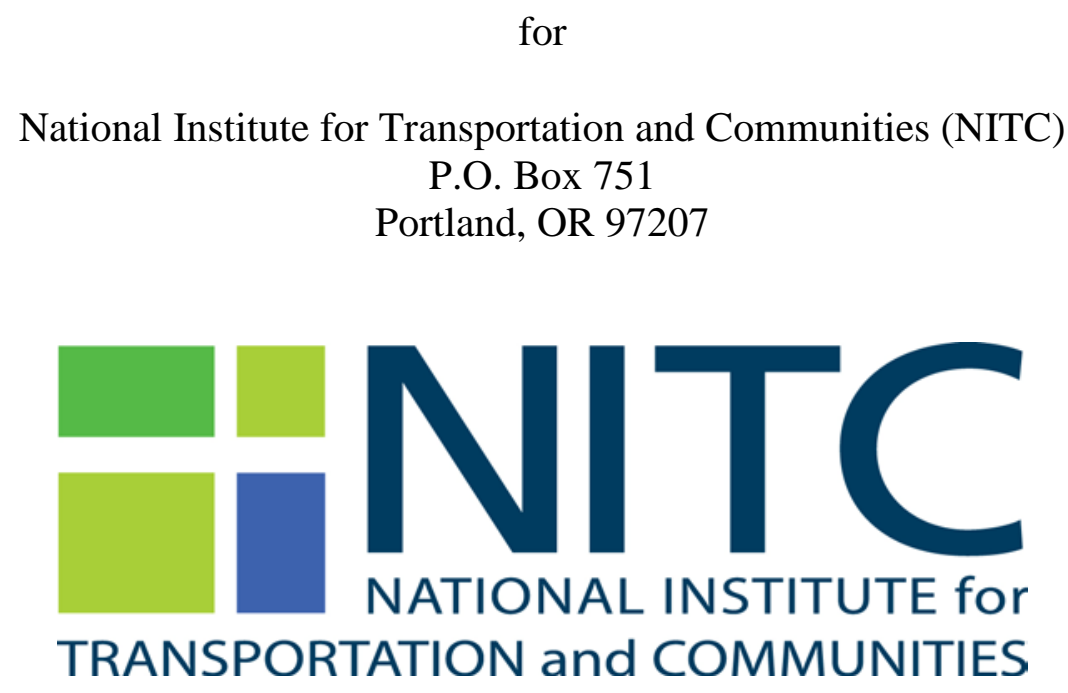

December 2018 


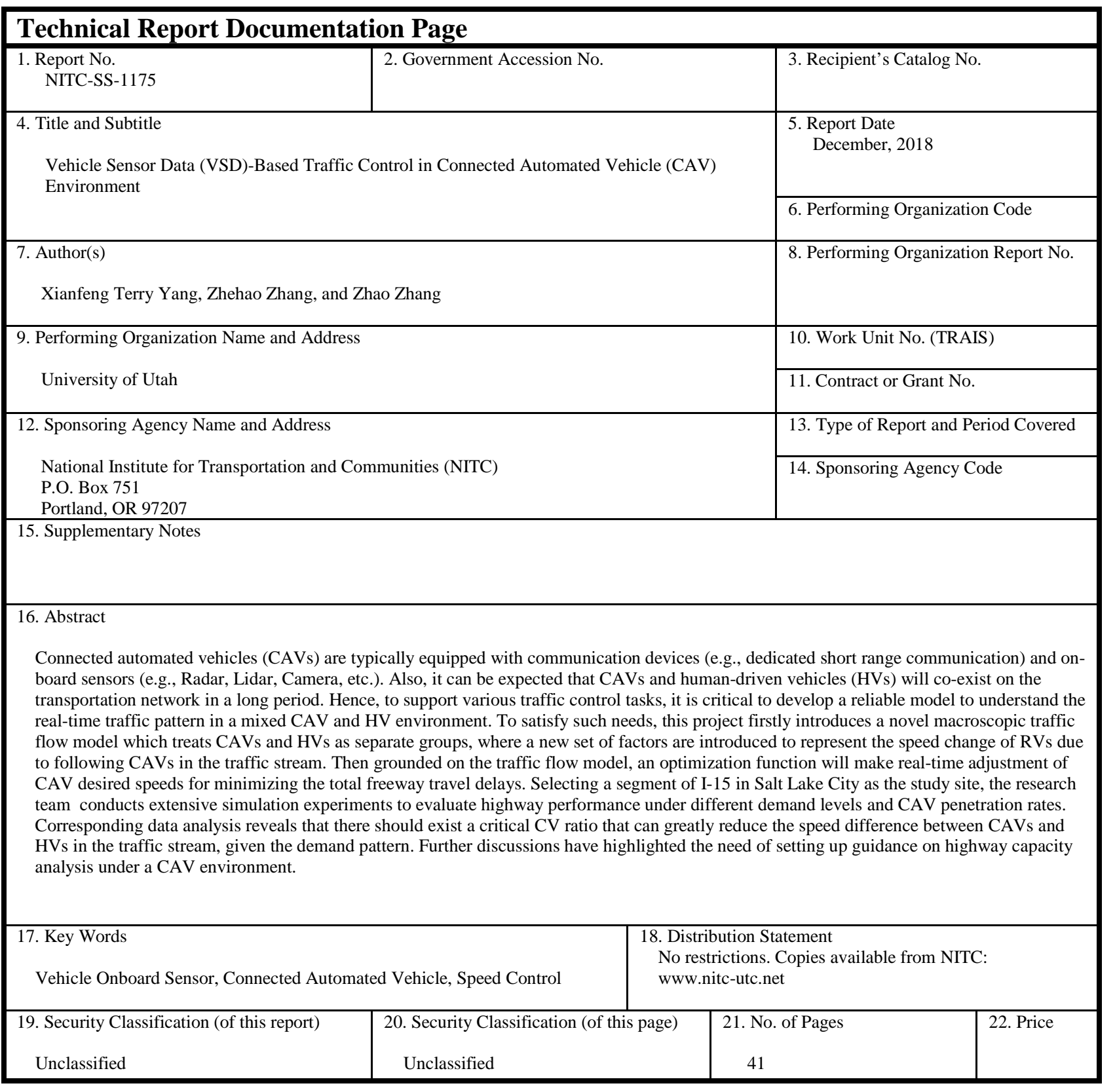




\section{ACKNOWLEDGEMENTS}

This project was funded by the National Institute for Transportation and Communities (NITC) under grant number (NITC-SS-1175). The PI Xianfeng Yang would also like to acknowledge the data support from the Utah Department of Transportation (UDOT).

\section{DISCLAIMER}

The contents of this report reflect the views of the authors, who are solely responsible for the facts and the accuracy of the material and information presented herein. This document is disseminated under the sponsorship of the U.S. Department of Transportation University Transportation Centers Program in the interest of information exchange. The U.S. Government assumes no liability for the contents or use thereof. The contents do not necessarily reflect the official views of the U.S. Government. This report does not constitute a standard, specification, or regulation.

\section{RECOMMENDED CITATION}

Yang, Xianfeng, Zhang, Zhehao and Zhang, Zhao. Vehicle Sensor Data (VSD) Based Traffic Control in Connected Automated Vehicle (CAV) Environment. NITC-SS-1175. Portland, OR: Transportation Research and Education Center (TREC), 2018. 


\section{TABLE OF CONTENTS}

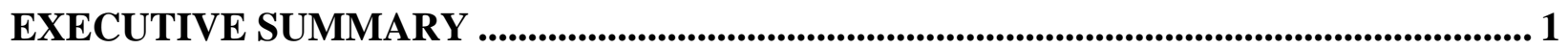

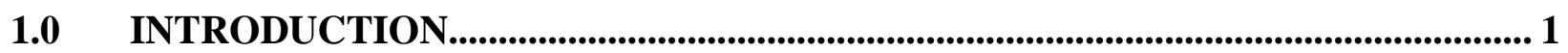

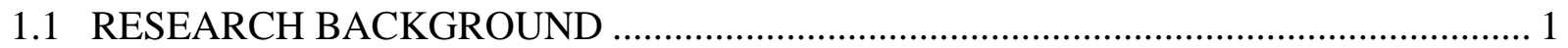

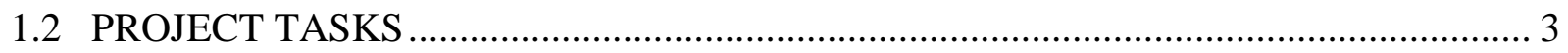

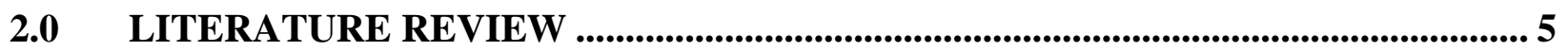

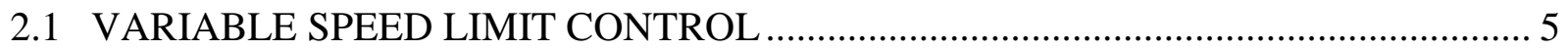

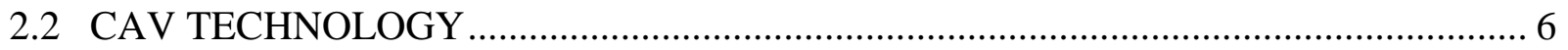

2.3 TRAFFIC CONTROL APPLICATIONS WITH CAV ................................................... 7

3.0 METHODOLOGY …........................................................................................ 8

3.1 CONTROL FRAMEWORK................................................................................... 8

3.2 TRAFFIC STATE ESTIMATION ……………………………………………........ 9

3.2.1 Macroscopic Traffic Flow Model - No CAV Involved............................................ 9

3.2.2 Mixed Traffic Flow Formulations ........................................................................ 10

3.3 CAV DESIRE SPEED OPTIMIZATION …………............................................... 12

3.3.1 Optimization Model with Prediction …………........................................................ 12

3.3.2 Rolling Horizon Control Logic .......................................................................... 13

3.4 HIGHWAY CAPACITY UNDER CAV IMPACT...................................................... 14

4.0 EXPERIMENTAL TESTS .................................................................................. 16

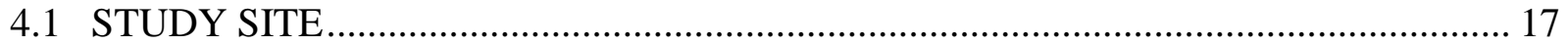

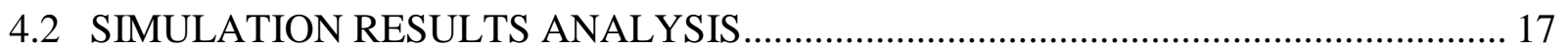

4.3 ANALYSIS CAV IMPACT TO HIGHWAY CAPACITY …….................................... 30

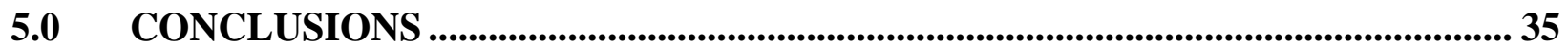

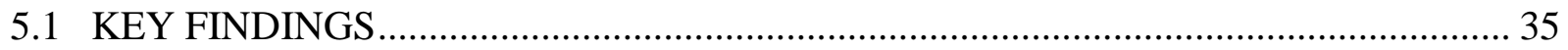

5.2 FUTURE RESEARCH DIRECTIONS ..................................................................... 36

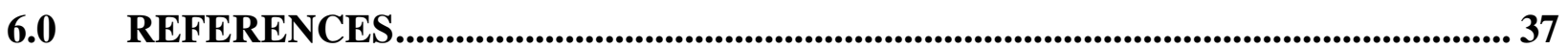

\section{LIST OF TABLES}

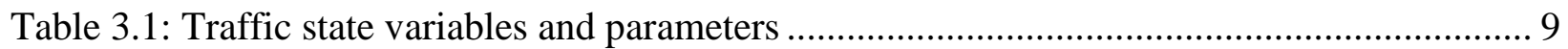

Table 3.2: Traffic state variables and parameters for mixed flow formulation ............................... 10

Table 3.3: Control variables for constraints................................................................................ 13

Table 4.1: Average delay during time periods with different ratio of connected vehicles in mixed flow

Table 4.2: Percentage improvement on average delay comparing CAV controls with no CAV controls. 29

Table 4.3: Mixed traffic flow simulation scenarios ....................................................................... 30

Table 4.3: The simulation results of the scenarios with critical CV ratio....................................... 31 


\section{LIST OF FIGURES}

Figure 1.1: Time-dependent speed distribution of vehicles.................................................. 2

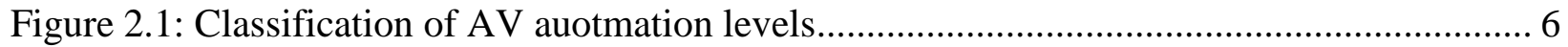

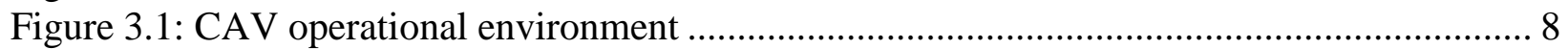

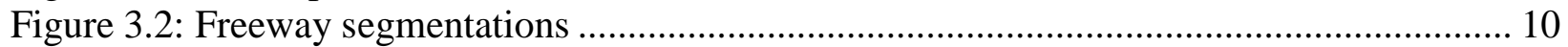

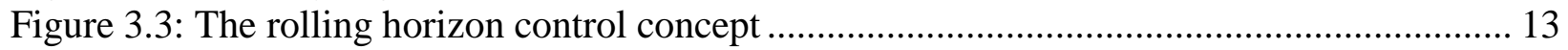

Figure 3.4: Inter-vehicle spacing in traffic mixed with CAVs and HVs ................................... 15

Figure 3.5: Fundamental diagram with RV only and mixed with CV and HV ......................... 15

Figure 3.6: Fundamental diagram with RV only and mixed with CV and HV ......................... 16

Figure 3.7: Fundamental diagram with RV only and mixed with CV and HV ........................ 16

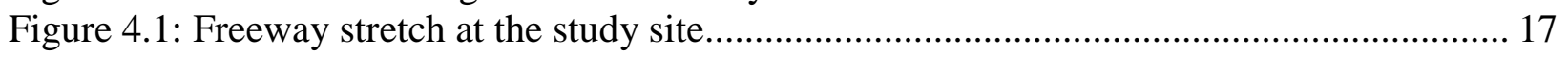

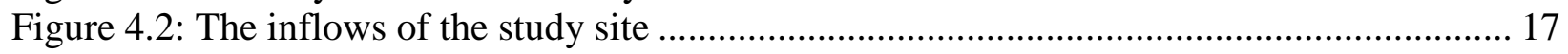

Figure 4.3: Mean speed with 20percent of connected vehicles in mixed flow ........................... 19

Figure 4.4: Mean speed with 50percent of connected vehicles in mixed flow ............................ 20

Figure 4.5: Mean speed with 70percent of connected vehicles in mixed flow........................... 22

Figure 4.6: Mean speed with 100percent of connected vehicles in mixed flow .......................... 23

Figure 4.7: Density with 20percent of connected vehicles in mixed flow................................. 25

Figure 4.8: Density with 50percent of connected vehicles in mixed flow................................ 26

Figure 4.9: Density with 70percent of connected vehicles in mixed flow................................. 27

Figure 4.10: Density with 100percent of connected vehicles in mixed flow............................ 29

Figure 4.11: The distribution of $\beta \mathrm{i}$ in different scenarios .................................................... 30

Figure 4.12: Freeway average travel time under different scenarios...................................... 31

Figure 4.13: Average travel time comparison between different vehicle groups ....................... 32

Figure 4.14: Reduced average travel time under different scenarios and critical CV rates.......... 33

Figure 4.15: Time-dependent travel time under different levels of demand ............................. 34 



\section{EXECUTIVE SUMMARY}

Connected automated vehicles (CAVs) are typically equipped with communication devices (e.g., dedicated short range communications (DSRC)) and on-board sensors (e.g., Radar, Lidar, Camera, etc.). Communication devices would enable the exchange of real-time information between vehicles and infrastructures via vehicle-to-vehicle (V2V) and vehicle-to-infrastructure (V2I) channels. Sensors equipped in vehicles are providing various vehicle sensor data (VSD) such as the CAV's GPS location, speed and moving direction (trajectory). Existing studies have shown the effectiveness of using CAV trajectories as input in many traffic control models.

However, it can be expected that CAVs and human-driven vehicles (HVs) will co-exist on the transportation network in a long period. Hence, to support various traffic control tasks, it is critical to develop a reliable model to understand the real-time traffic pattern in a mixed CAV and HV environment. To satisfy such needs, this project firstly introduces a novel macroscopic traffic flow model which treats CAVs and HVs as separate groups, where a new set of factors are introduced to represent the speed change of HVs due to following CAVs in the traffic stream. Then grounded on the traffic flow model, an optimization function will make real-time adjustment of CAVs' desired speeds for minimizing the total freeway travel delays.

Selecting a segment of I-15 in Salt Lake City as the study site, the research team conducts extensive simulation experiments to evaluate highway performance under different demand levels and CAV penetration rates. Corresponding data analysis reveals that there should exist a critical CAV ratio that can greatly reduce the speed difference between CAVs and HVs in the traffic stream, given the demand pattern. Further discussions have highlighted the need of setting up guidance on highway capacity analysis under the CAV environment.

\subsection{INTRODUCTION}

\subsection{RESEARCH BACKGROUND}

By exchanging real-time information between vehicles and infrastructures, CV applications have demonstrated significant benefits on improving the safety and mobility of transportation and reducing emissions. Along another track, current autonomous vehicles (AVs) mostly rely on different types of sensors. Ultrasonic, radar and camera technologies allow AVs to observe and analyze their surroundings and to automatically take suitable driving behaviors (e.g., deceleration, acceleration, lane changing, etc.). When connectivity is added into the AV-based system, the vehicles become connected and automated vehicles (CAVs) which are equipped with both on-board units (OBUs) for communications and sensors for detection. Specifically, the 
added V2V technology allows CAVs to exchange critical vehicle status data such as vehicle speeds, location and acceleration . and the V2I platform supports vehicles' communications with infrastructure (e.g., receives signal phase and timing data from signal controller). Since CV technology brings unparalleled benefits, many researchers have investigated its application in various traffic control domains. In addition, some transportation agencies have started to deploy $\mathrm{CV}$ technologies in real-world applications during the past few years. However, it may take a long period to reach a fully CVs environment and CVs must co-exist with human-driven vehicles (HVs) on the roads at the current stage. Hence, to support many traffic control tasks, it is critical to develop a reliable traffic state estimation model that can fully capture the mixed CAV and HV traffic pattern.

Traffic state estimation has long been identified as an important task within a traffic control loop. Freeway traffic state estimation refers to estimating all variables of traffic flow at the current time instant based on real-time traffic measurements. A reliable traffic flow estimation would be the foundation of good traffic management strategies. Since speed control is often applied to CAVs in most applications, changing of CAV motion states would inevitably affect the speed of the whole traffic stream as CAVs and HVs are sharing the roads. Figure 1 shows the vehicle speed distribution under different two simulation cases. Notably, case 1 was simulated with mixed traffic and CAVs are operated with advisory speeds (slightly lower than the average traffic speed), while case 2 was simulated with HVs only. The resulting comparisons indicate that despite speed control is only performed on CAVs, the average speed of HVs was also reduced. Hence, one of the most challenging issues in traffic state estimation will be how to effectively capture the interactions between CAVs and HVs under the mixed traffic environment. To address such needs, this project aims to develop a novel modeling approach by treating them as separate groups. In addition, a new set of factors is introduced to represent the speed change of HVs due to following CVs in the traffic stream. Then an optimization function, grounded on the traffic flow model, will be implemented to determine the optimal desired speeds of CAVs in the next control period, with the objective of minimizing total freeway travel time.

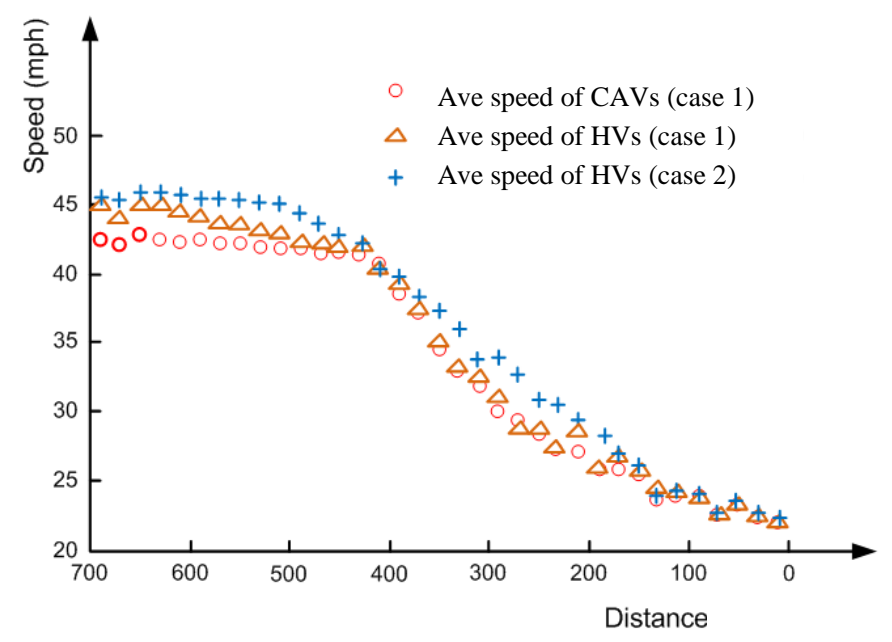

Figure 1.1: Time-dependent speed distribution of vehicles

In summary, emerging CAV technologies offer the potential to utilize the real-time roadway traffic information to improve traffic safety, efficiency and stability. These benefits of CV 
technologies are usually achieved by optimal CAV speed control. In the control loop, infrastructures provide advisory speeds to CAVs to follow and CAVs send their trajectory data and safety information to infrastructures for future feedback operations. However, despite some existing studies that proved the effectiveness of improving highway capacity performance by optimally controlling CAVs, its penetration rate plays a key role in affecting the system's performance. This is explicated by how HV drivers would respond to the speed change of their nearby CAVs. Grounded in preliminary simulation studies, it can be observed that HVs will follow CAVs in the traffic stream when they have no sufficient gap to make lane changes (e.g., under congested conditions or with a high CAV penetration rate). In such cases, the control of CAVs will concurrently affect HVs' speeds. However, under the conditions of light traffic or low CAV rate, HVs can easily change lanes to avoid following CAVs. It may even increase the potential crash rate due to increased lane-changing maneuvers. Therefore, given a congestion level, there should exist a critical CAV penetration rate for each highway segment specified with its capacity. If the CAV rate can be above the critical value, the effectiveness of CAV-based control algorithms will be maximized. Grounded in the developed CV optimal speed control model, this paper further explores the existence of a critical CV penetration rate and study its impact on highway capacity.

\subsection{PROJECT TASKS}

This project includes the following five key tasks:

- Literature review, where our research team has conducted comprehensive reviews on research in various domains such as variable speed limit control, CAV technology development, and traffic control applications under a CAV environment.

- Traffic state estimation model development, where a macroscopic traffic flow model is formulated to capture the speed interactions between CAVs and HVs in the mixed traffic pattern.

- CAV desired speed optimization model development, where a rolling horizon control concept is implemented to optimize CAVs' desired speed in the following control period, using the objective of minimizing total freeway travel time.

- Highway capacity analysis under a CAV environment, where we further examine the change of highway performance under different congestion levels and explore the sensitivity of impacts by the CAV penetration rate.

- Experimental tests, where all proposed control models are tested on a selected study site, a freeway segment on I-15 in Salt Lake City. 



\subsection{LITERATURE REVIEW}

\subsection{VARIABLE SPEED LIMIT CONTROL}

Despite many recent research efforts that have been attracted to CAV-related optimal speed control, a similar concept but on HVs only, named variable speed limit (VSL) control, has already been studied extensively in the literature. VSL is initially designed to reduce the speed difference on some hazardous highway segments so as to decrease the rear-end collision rate and improve traffic safety (Anund et al., 2009). Recently, it has been recognized that VSL may offer the potential to mitigate traffic congestion and improve traffic efficiency at work zones and freeway bottlenecks. Through dynamically changed speed limits along a controlled segment, VSL can smooth the speed transition between the upstream and congested downstream flows, and minimize the impact of shockwave on traffic conditions. The mitigation of traffic speed variance can facilitate traffic flows to better utilize the available roadway capacity during congestion periods.

Mainly for the work-zone safety concerns, a set of studies (Lyles et al., 2003; Lin et al., 2004; Kang, 2006; Kwan et al., 2015; Yang et al., 2016; Lin et al., 2018) has been conducted to explore the potential of improving traffic efficiency with the VSL control. Those preliminary but promising results have motivated more researchers to investigate the applicability of using VSL for recurrently congested roadway segments. Despite the potential benefits of VSL for workzone operations, design of reliable algorithms to ensure its benefits in contending with recurrent congestion remains a challenging issue. For example, the Dutch VSL experiment (Smulders, 1990) showed no improvement in capacity which may be attributed to its advisory purpose. More recently, Chang et al. (2011) reported a successful implementation of an integrated VSL and travel time information system on MD 100 near Coca-Cola Drive, which achieved travel time and throughput improvements.

Along the same line, a study on the I-495 Capital Beltway (Sisiopiku et al., 2009) revealed that VSL can delay the formation of bottleneck congestion. Abdel-Aty et al. (2008) developed a VSL system for I-4 through Orlando, FL, that was reported to reduce both crash risk and travel time under a simulated environment. Hegyi et al. (2006) modified the METANET macroscopic traffic flow model to incorporate the VSL effect and adopted the model predictive control (MPC) approach to determine the optimal speed limit. Papageorgiou et al. (2008) and Carlson et al. (2010) analyzed the effect of VSL on aggregated traffic flow behavior from the theoretical perspective, and proposed an open-loop integrated optimal control framework to coordinate ramp metering with VSL control. Their simulation results show a reduction of 15 percent in total travel time. Most recently, Hadiuzzaman and Qiu (2012) proposed a modified CTM-based VSL control, and also used the MPC method to dynamically change the speed limit in real time. Similar logic has been implemented by Yang et al. (2015), which uses the Extended Kalman Filter for estimation correction. 


\subsection{CAV TECHNOLOGY}

Reliable and seamless V2V and V2I data communication is the critical component of CV technology applications (Dey et al., 2016). Various wireless technologies have been used to support the data transfer requirement of diverse ITS applications, such as Bluetooth, ZigBee, Passive RFID, Ultra Wide Bandwidth (UWB) and mmWave communications (Lu et al., 2014). The selection of a wireless communication option relies on the accessibility and feasibility of wired and wireless communication options and data transfer requirement of particular applications. While existing ITS applications are infrastructure-based (i.e., installed at the roadside locations) (Silva et al., 2017), the next major deployment of wireless technologies within the transportation grid is the high-speed wireless communication between moving vehicles and transportation infrastructure.

To meet the requirements of CV applications (e.g., fast acquisition, low latency with high reliability, highest security and privacy standards), the Federal Communications Commission had previously allocated the $5.9 \mathrm{GHz}$ band $(5.85 \mathrm{GHz}$ to $5.925 \mathrm{GHz})$ for V2V/V2I communications, also known as dedicated short range communications (DSRC) (Kenney, 2011; Xu et al., 2004; Jiang et al., 2006; Yin et al., 2004; Taliwal et al., 2004). These communications include that of safety messaging, geographic locations, sensor data (e.g., tire pressure), which involves periodic broadcast of time-sensitive status information by vehicles (Bai et al., 2010; Oh et al., 1999; Wang et al., 2008; Ma et al., 2009).

On the other hand, AVs are operated with the support of various vehicle automation functions, which rely on real-time sensor data for making maneuver decisions (e.g., acceleration, deceleration, lane changes). In current applications three types of sensors, including cameras, radars and LIDARs, are commonly used for detections (Huang et al., 2018). According to the capability of SAE, vehicle automation functions have been categorized into six levels (Andersen, 2017), as shown in Figure 2.1.

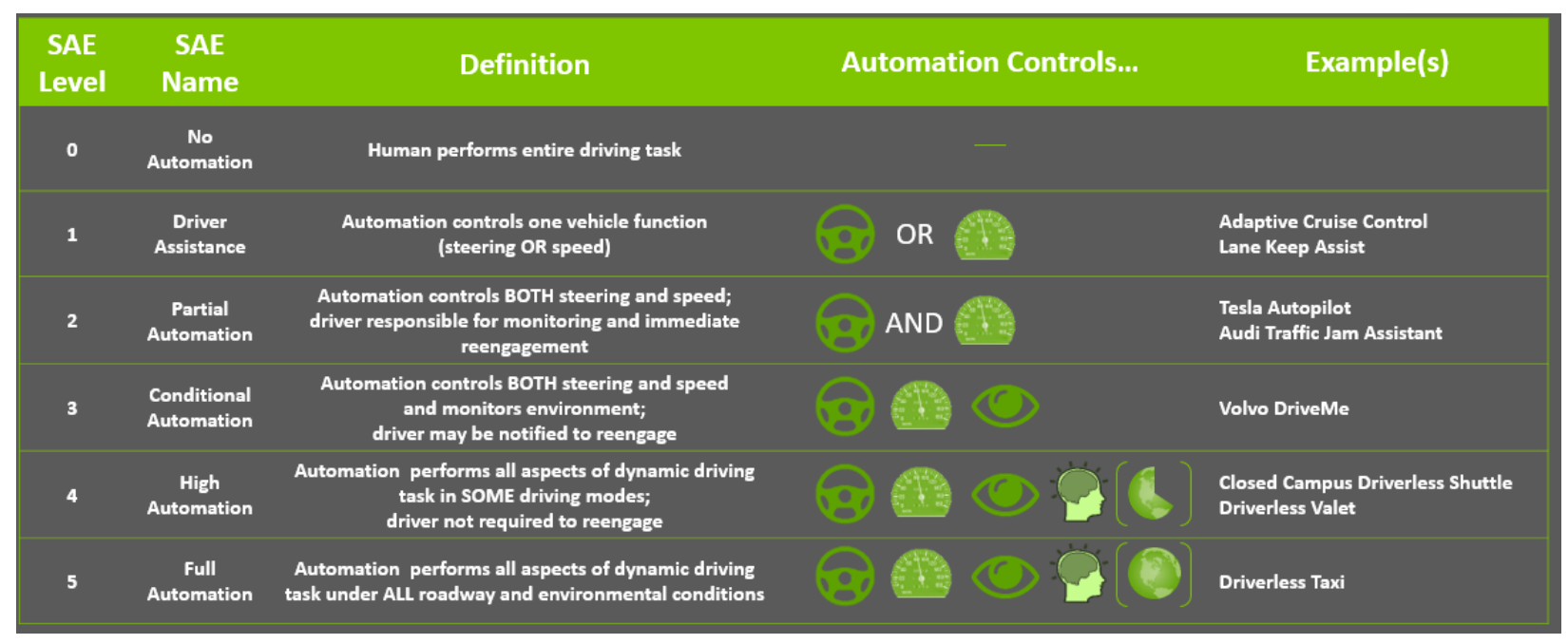

Figure 2.1: Classification of AV auotmation levels 
In summary, the vehicular network combines wireless communication with sensing devices installed on vehicles. With in-vehicle sensing technologies, CAV-based systems are capable of collecting abundant driving data, such as speed and engine parameters, from a large number of vehicles. Such data are characterized as large volume, multifrequency, and multisource, which largely reflect the vehicle and the road traffic status and thereby are widely used to adjust CAVs' speed by automation functions.

\subsection{TRAFFIC CONTROL APPLICATIONS WITH CAV}

CAV technology has the potential to enable precise control of individual vehicle trajectories (Ahn et al., 2013; Wang et al., 2014; Huang et al., 2018). With such trajectory control, vehicles can either adjust their driving behavior (e.g., speed, deceleration, acceleration) based on the approaching traffic signal timing plan (Kamalanathsharma et al., 2013) or coordinate with other vehicles to pass through an intersection during a green light (Dresner and Stone, 2008; Lee and Park, 2012). Notably, the car-following models under a CAV environment are commonly treated as the foundation of developing CAV traffic control systems. A group of studies, from the system control point of view, have contributed various methods to making CAV-based control more available for field deployments. Representatives of those include frequency-domain methods (Cook, 2007; Monteil et al., 2014), linear robust control theories (Hao and Barooah, 2013; Lin et al., 2012), and Lyapuno theory (Oncu et al., 2014).

To characterize CAVs' behaviors and their potential impact on traffic streams, existing studies can be categorized into two types of efforts (Wei et al., 2017). The first type of studies (Horowitz and Varaiya, 2000; Gong et al., 2016) focus on understanding interactions between CAVs and regular vehicles by examining how CAV dynamics can change traffic characteristics. This is induced by the significantly different driving behaviors between humans and vehicle automation functions. The second type aims to evaluate the change of roadway capacity due to CAVs deployment (Ghiasi et al., 2017). For example, shorter CAV reaction time would allow closer spacing between cars and the roadway capacity could be consequently increased (Bose and Ioannou, 1999). More recently, based on the simulation of a network with 16 intersections, Lioris et al. (2017) have proved that platooning of CAVs can even double the throughput on the roads.

Quite a few existing studies focused on addressing the individual vehicle trajectory control (He et al., 2015; Wu et al., 2015;). Dynamic programming (DP)-based algorithm lays the theoretical foundation for single-vehicle trajectory optimization. However, DP-based trajectory optimization is oftentimes inefficient for real-world application due to its computational complexity, let alone applying to the scenarios involving multiple-vehicle trajectories control.

To fill this research gap, Flint et al. (2002) proposed an approximate DP algorithm for multiple AVs to move cooperatively. McNaughton (2011) developed a five-dimensional search space formulation for AV motion planning. More recently, Zhou et al. (2017) presented a parsimonious shooting heuristic $(\mathrm{SH})$ algorithm that can effectively smooth trajectories of a stream of CAVs approaching a signalized intersection. A time geography-oriented approach is combined with Newell's simplified car-following model to control a detailed acceleration profile. Other studies also incorporated Newell's simplified car-following model for CAV platooning strategy (Bang and Ahn, 2017). Following their preceding study (Zhou et al., 2017), Ma et al. (2017) discussed 
the SH algorithm on optimality and computational complexity. They proposed a framework for CAVs under centralized control for multitrajectory optimization with only a few control variables. Wei et al. (2017) developed mathematically rigorous optimization models and computationally tractable algorithms to model the dynamic process of tight platoon formation and system-level control for AVs. The vehicle trajectories are optimized subject to minimal safe driving distance between cars, as well as different entrance and exit boundary conditions.

\subsection{METHODOLOGY}

\subsection{CONTROL FRAMEWORK}

The connectivity technology of CAVs allows the exchange of traffic information between vehicles and infrastructure. The vehicle automation function will automatically adjust the speed in real time according to the desired travel speed and distance to the vehicle ahead. As shown in Figure. 3.1, roadside traffic sensors can collect the traffic data such as flow rate (number of vehicles) and average vehicle speed. Through the communication infrastructure, data from multiple neighboring sensors will be recorded and sent to CAVs (V2I). Then the computational devices on CAVs can perform computational works based on the real-time data. For the need of vehicle automation, it always requires reliable sensors (e.g., LiDAR, camera) on automated vehicles. With the integration and coordination of different types of sensors, the automated vehicles can be aware of the locations and speeds of surrounding vehicles. Such detected information can be further sent to other nearby CAVs through the V2V communication platform.

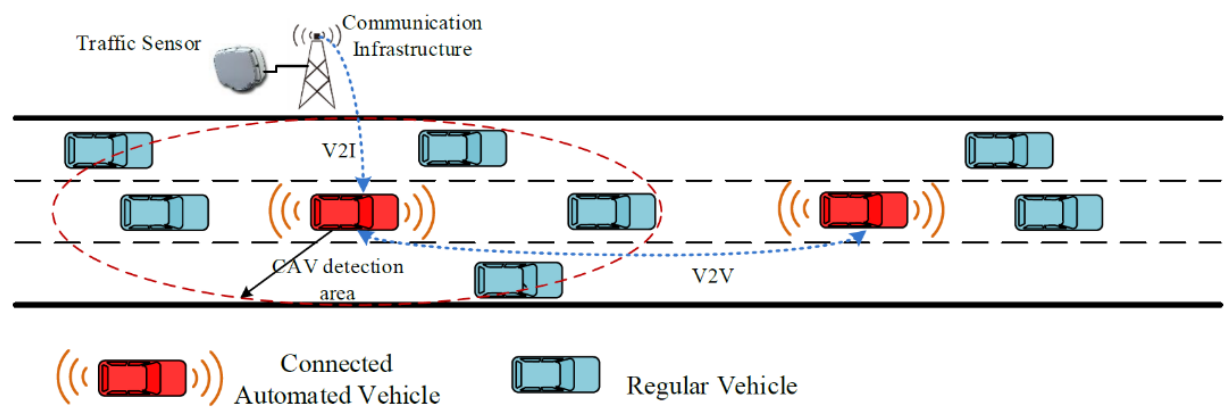

Figure 3.1: CAV operational environment

Using the real-time CAV trajectory data and roadside sensor data as input, the operational structure of the proposed vehicle sensor data (VSD)-based CAV speed control system includes the following three principal components:

1) Traffic state model: Given the upstream flow rate, the on-ramp and off-ramp flow rate, the model will function to predict the traffic state evolution in each freeway subsection for the case of no-CAV involved. An optimization model grounded in such a model will serve as a comparison for evaluating the effectiveness of the proposed CAV control system. 
2) Mixed traffic flow model: This new model aims to capture the speed impact of CAVs on HVs in mixed flow. The model is an extension of a conventional traffic state model by dividing CAVs and HVs as separate groups.

3) Optimization model: Based on the estimated conditions from an embedded traffic flow model, the system will execute the optimization model to predict the traffic state in the next prediction horizon and yield optimal desired speeds of CAVs.

Notably, the proposed framework is based on an assumption that CAVs would be operated with suggested desired speeds and HVs are sharing road lanes with CAVs in the traffic stream. Based on the optimal desired speed profile along the freeway, CAVs would make real-time adjustments according to their distance to nearby vehicles.

\subsection{TRAFFIC STATE ESTIMATION}

\subsubsection{Macroscopic Traffic Flow Model - No CAV Involved}

As shown in Figure. 3.2, the target freeway segment is conceptually divided into $\mathrm{N}$ subsections with a unit length of $\Delta \mathrm{L}$. Notably, the $\Delta \mathrm{L}$ should be sufficiently long so that vehicles cannot pass one subsection during one-time interval k. Moreover, each subsection can have at most one onramp and one off-ramp. For convenience of discussion, variables and parameters used in this model are listed in the following table:

Table 3.1: Traffic state variables and parameters

\begin{tabular}{l|l}
\hline Variables and parameters & Definitions \\
\hline $\mathrm{q}_{\mathrm{i}}(\mathrm{k})$ & Transition flow rate entering segment (i+1) from segment i during interval $\mathrm{k} ;$ \\
\hline $\mathrm{r}_{\mathrm{i}}(\mathrm{k})$ & On-ramp flow rate entering segment i during interval $\mathrm{k} ;$ \\
\hline $\mathrm{s}_{\mathrm{i}}(\mathrm{k})$ & Off-ramp flow rate leaving segment i during interval $\mathrm{k} ;$ \\
\hline $\mathrm{d}_{\mathrm{i}}(\mathrm{k})$ & Mean traffic density per lane in segment i during interval $\mathrm{k} ;$ \\
\hline $\mathrm{u}_{\mathrm{i}}(\mathrm{k})$ & Mean speed in segment i during interval $\mathrm{k} ;$ \\
\hline$\gamma, \tau, \kappa, a$ & Traffic state model parameters; \\
\hline$\Delta \mathrm{L}$ & Length of each freeway segment; \\
\hline$\lambda_{\mathrm{i}}$ & Number of lanes in subsection $\mathrm{i} ;$ \\
\hline
\end{tabular}

For each subsection $\mathrm{i}$, the mean density, $\mathrm{d}_{\mathrm{j}}(\mathrm{k})$, can be determined by the difference between the input and output flows as follows:

$$
d_{i}(k+1)=d_{i}(k)+\frac{\Delta T}{\lambda_{i} \Delta L}\left[q_{i-1}(k)-q_{i}(k)+r_{i}(k)-s_{i}(k)\right]
$$




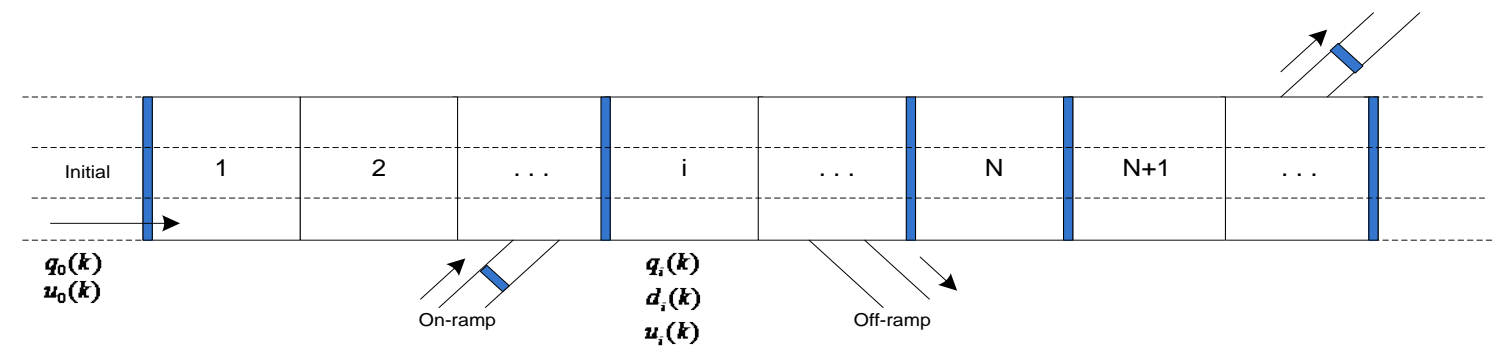

Figure 3.2: Freeway segmentations

For dynamically updating the average speed, $\mathrm{u}_{\mathrm{i}}(\mathrm{k})$, a well-developed equation proposed by the METANET model is adopted and shown as follows:

$$
\begin{gathered}
u_{i}(k+1)=u_{i}(k)+\frac{\Delta T}{\tau_{i}}\left[V_{i}\left\{d_{i}(k)\right\}-u_{i}(k)\right]+\frac{\Delta T}{L_{i}} u_{i}(k)\left[u_{i-1}(k)-u_{i}(k)\right] \\
-\frac{\gamma_{i} \Delta T}{\tau_{i} \Delta L} \frac{\left[d_{i+1}(k)-d_{i}(k)\right]}{\left[d_{i}(k)+\kappa\right]}
\end{gathered}
$$

where, $V\left[d_{i}(k)\right]$ is the static speed for segment $i$ at time $k$ with respect to the density $d_{i}(k)$ :

$$
V\left[d_{i}(k)\right]=u_{f} \exp \left[-\frac{1}{a}\left(\frac{d_{i}(k)}{d_{c r}}\right)^{a}\right]
$$

Also, the relationship between flow, density and speed is given by the following:

$$
q_{i}(k)=d_{i}(k) u_{i}(k) \lambda_{i}
$$

Using the inflow rate that can be collected by detectors installed at the upstream segment and onramps, one can directly use Equations 1-4 to estimate and predict the traffic state evolution on the target freeway segment.

\subsubsection{Mixed Traffic Flow Formulations}

Under the mixed CAV and HV environment, the proposed system would yield optimal desired speeds to CAVs through the I2V communication platform. As CAVs and HVs are sharing the roadways, it can be expected that the speed change of CAVs would inevitably affect the speeds of nearby HVs, which is usually called "CAV impact." Notably, such an impact would be quite sensitive to the traffic congestion level and CAV penetration rate. However, modeling the interactions between CAV and $\mathrm{HV}$ is still an unknown territory and the conventional traffic state model which treats all vehicles as a group may fall short of accuracy.

In this project, the proposed system utilized a novel mixed traffic state model which accounts for two different vehicle classes (CAVs and HVs) during the operational period. For convenience of discussion, variables and parameters used in this model are listed in the following table:

Table 3.2: Traffic state variables and parameters for mixed flow formulation 


\begin{tabular}{|c|c|}
\hline Variables and parameters & Definitions \\
\hline $\mathrm{d}_{\mathrm{j}, \mathrm{i}}(\mathrm{k})$ & The density of class $j$ vehicles for segment $i$ at time step $k$; \\
\hline $\mathrm{u}_{\mathrm{j}, \mathrm{i}}(\mathrm{k})$ & The mean speed of class j vehicles for segment i at time step k; \\
\hline $\mathrm{V}_{\mathrm{j}}\left[\mathrm{d}_{\mathrm{j}, \mathrm{i}}(\mathrm{k})\right]$ & $\begin{array}{l}\text { The static speed of class j vehicles for segment } \mathrm{i} \text { at time } \mathrm{k} \text { with respect to the } \\
\text { density of class j vehicles; }\end{array}$ \\
\hline $\mathrm{q}_{\mathrm{j}, \mathrm{i}, \mathrm{out}}(\mathrm{k})$ & $\begin{array}{l}\text { The flow rate of class } j \text { vehicles leaving segment } \mathrm{i} \text { to downstream segment } \\
\mathrm{i}+1 \text { between steps }(\mathrm{k}, \mathrm{k}+1) \text {; }\end{array}$ \\
\hline $\mathrm{q}_{\mathrm{j}, \mathrm{i}, \mathrm{in}}(\mathrm{k})$ & $\begin{array}{l}\text { The flow rate of class } \mathrm{j} \text { vehicles entering segment } \mathrm{i} \text { from upstream segment } \mathrm{i} \text { - } \\
1 \text { between steps }(\mathrm{k}, \mathrm{k}+1) \text {; }\end{array}$ \\
\hline $\mathrm{r}_{\mathrm{j}, \mathrm{i}}(\mathrm{k})$ & $\begin{array}{l}\text { The flow rate of class } \mathrm{j} \text { vehicles entering segment i from on-ramps between } \\
\text { steps }(\mathrm{k}, \mathrm{k}+1) \text {; }\end{array}$ \\
\hline $\mathrm{s}_{\mathrm{j}, \mathrm{i}}(\mathrm{k})$ & $\begin{array}{l}\text { The flow rate of class j vehicles leaving segment i from off-ramps between } \\
\text { steps }(k, k+1) \text {; }\end{array}$ \\
\hline $\mathrm{L}_{\mathrm{i}}$ & The length of segment i; \\
\hline$\lambda_{\mathrm{i}}$ & The number of lanes of segment i; \\
\hline$\Delta \mathrm{T}$ & The length of update time interval; \\
\hline $\mathrm{u}_{\mathrm{f}, \mathrm{ji}}$ & The free-flow speed of segment i for class j vehicles; \\
\hline $\mathrm{d}_{\mathrm{cr}, \mathrm{ji}}$ & The critical density of segment i for class $\mathrm{j}$ vehicles; \\
\hline $\mathrm{a}_{\mathrm{ji}}$ & The speed exponent term of segment i for class j vehicles; \\
\hline$\tau_{\mathrm{i},}, \gamma_{\mathrm{i}}, \kappa_{\mathrm{i}}, \beta_{\mathrm{i}}$ & The parameters in the dynamic speed equations of segment i; \\
\hline
\end{tabular}

Following the same notion of the conventional traffic flow model, the mixed traffic flow model can be expressed as follows:

$$
\begin{gathered}
d_{j, i}(k+1)=d_{j, i}(k)+\frac{\Delta T}{L_{i} \lambda_{i}}\left[q_{j, i, i n}(k)-q_{j, i, \text { out }}(k)+r_{j, i}(k)-s_{j, i}(k)\right] \\
q_{j, i, i n}(k)=q_{j, i-1, \text { out }}(k) \\
V_{j, i}\left[d_{j, i}(k)\right]=u_{f, j i} \exp \left[-\frac{1}{a_{j i}}\left(\frac{d_{1, i}(k)+d_{2, i}(k)}{d_{c r, j i}}\right)^{a_{j i}}\right] \\
u_{1, i}(k+1)=u(k)+\frac{\Delta T}{\tau_{i}}\left[V_{1, i}(k)-u_{1, i}(k)\right]+\frac{\Delta T}{L_{i}} u_{1, i}(k)\left[u_{1, i-1}(k)-u_{1, i}(k)\right] \\
-\frac{\gamma_{i} \Delta T}{\tau_{i} L_{i}} \frac{\left[d_{1, i+1}(k)+d_{2, i+1}(k)-d_{1, i}(k)-d_{2, i}(k)\right]}{\left[d_{1, i}(k)+d_{2, i}(k)\right]}-\frac{\beta_{i} \Delta T}{\tau_{i}}\left[V_{1, i}(k)-V_{2, i}(k)\right] \\
u_{2, i}(k+1)=u_{2, i}(k)+\frac{\Delta T}{\tau_{i}}\left[V_{2, i}(k)-u_{2, i}(k)\right]+\frac{\Delta T}{L_{i}} u_{2, i}(k)\left[u_{2, i-1}(k)-u_{2, i}(k)\right] \\
-\frac{\gamma_{i} \Delta T}{\tau_{i} L_{i}} \frac{\left[d_{1, i+1}(k)+d_{2, i+1}(k)-d_{1, i}(k)-d_{2, i}(k)\right]}{\left[d_{1, i}(k)+d_{2, i}(k)\right]} \\
q_{j, i}(k)=\lambda_{i} d_{j, i}(k) u_{j, i}(k)
\end{gathered}
$$


To reflect the interactions between CAVs and HVs, the term of speed difference is introduced into Eq. (3-8). Considering some HVs would follow CAVs in the traffic stream, the speed difference term can be simplified as a set of impact factors, $\beta(\mathrm{k})$. As discussed in previous sections, $\beta(\mathrm{k})$ shall be varied with the change in congestion level and CAV penetration rate. To calibrate the values of $\beta(\mathrm{k})$, one shall obtain the flows and mean speeds of all vehicles from road side traffic sensors and flows and mean speeds of CAVs based on their trajectories. In this project, due to the lack of field data, we adopted the values of $\beta(\mathrm{k})$ in $\mathrm{Lu}(2016)$ which are calibrated by extensive simulation experiments.

For each segment with CAVs involved, one can reconstruct Eq. (3-6) as follows to represent the flow of two different class of vehicles:

$$
\begin{gathered}
q_{1, i, \text { in }}(k)=\left(1-\alpha_{i}\right)\left[q_{1, i-1, \text { out }}(k)+q_{2, i-1, \text { out }}(k)\right] \\
q_{2, i, \text { in }}(k)=\alpha_{i}\left[q_{1, i-1, \text { out }}(k)+q_{2, i-1, \text { out }}(k)\right]
\end{gathered}
$$

\subsection{CAV DESIRE SPEED OPTIMIZATION}

\subsubsection{Optimization Model with Prediction}

Based on the traffic state at the current time interval, one can further implement the traffic flow model, Eqs. (3-5) (3-12), to predict the traffic state within the next prediction horizon with respect to different CAV desired speeds. Then, the one with the best objective output can be selected for implementation. Hence, the first step of the optimization model is to select a proper control objective function.

As the primary focus of CAV speed control is to reduce freeway travel time and increase flow stability, this project adopts the following objective of minimizing total travel time over the controlled segment:

$$
\min \sum \sum \lambda_{i}\left[d_{1, i}(k)+d_{2, i}(k)\right] \Delta T
$$

To implement the optimization model and select the proper control speed for the projected control period, one shall place the following additional constraints:

$$
\left\{\begin{array}{cc}
u_{j a m} \leq u_{j, i}(k) \leq u_{f, j i}, & \text { segment } i \text { without } C A V \text { invovled } \\
u_{j a m} \leq u_{j, i}(k) \leq u_{f, j i} v_{i}(k), & \text { segment } i \text { with } C A V
\end{array}\right.
$$

where,

$$
0<v_{i}(k) \leq 1
$$

One shall also set the density boundaries to reflect the jam density constraint as follows:

$$
0 \leq d_{j, i} \leq d_{j a m}
$$


Also, for safety concern, the speed variation between consecutive intervals shall be set within the following boundaries:

$$
-\delta \leq u_{f, j i} v_{i}(k)-u_{f, j i} v_{i}(k-1) \leq \delta
$$

Note that the speed variation between consecutive intervals shall be limited within a given range, such as $5 \mathrm{mi} / \mathrm{hr}$. In addition, considering the feasible solution set is quite small, one can adopt an enumerative search for obtaining the optimal solution. The control variables in the control model are listed in following table:

Table 3.3: Control variables for constraints

\begin{tabular}{l|l}
\hline Variables and parameters & Definitions \\
\hline $\mathrm{V}_{\mathrm{i}}(\mathrm{k})$ & VSL ratio in segment i during interval $\mathrm{k}$ \\
\hline $\mathrm{d}_{\mathrm{jam}}$ & Jam traffic density \\
\hline $\mathrm{d}_{\mathrm{c}}$ & Critical traffic density \\
\hline $\mathrm{u}_{\mathrm{f}}$ & Free flow speed \\
\hline$\Delta$ & Maximum allowable difference for VSL change \\
\hline
\end{tabular}

\subsubsection{Rolling Horizon Control Logic}

In the proposed optimization model, the generated CAV optimal desired speeds cannot be changed frequently, due to the consideration of traffic safety and HVs' reactions to the speed change. Therefore, a control horizon $\mathrm{T}^{\mathrm{C}}$ (e.g., $1 \mathrm{~min}$ ) is defined to remain the unchanged of CAV desired speeds. Also note that the optimization model will be activated once all data collected by CAVs and detectors have been updated. Hence, during each control horizon, the optimization model will produce multiple estimates of the optimal speed limit over a longer prediction horizon (e.g., 10 mins), and the process to select the robust one prior to its execution is shown in the succeeding text. Such rolling horizon control logic is illustrated in the following figure:

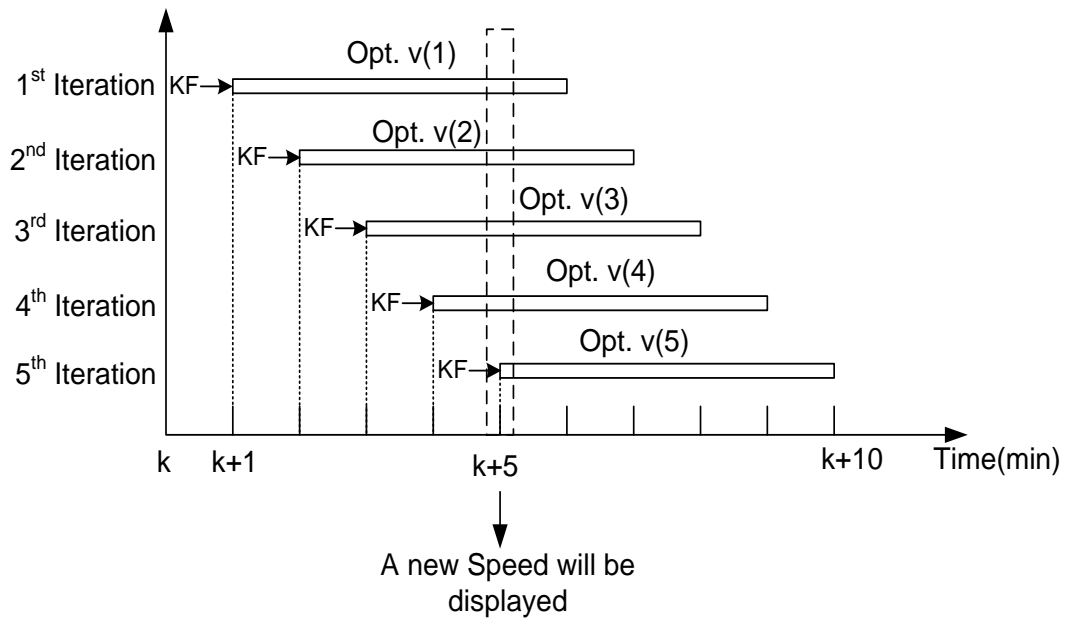

Figure 3.3: The rolling horizon control concept 
For example, assuming that the control horizon is set to be two minutes and the prediction horizon is set to be 10 minutes, the proposed model would project the resulting travel times over the next 10 minutes with respect to different CAV desired speed profile. After the optimal one is selected, it would be implemented for only two minutes.

Given the set of computed optimal speed limits for the same horizon, $\{v(1), v(2), \ldots, v(n)\}$, one can determine the speed limits to be displayed based on the following procedure:

- Define a counter $\mathrm{M}$ to identify the moving direction of the speed limit, and then denote $\mathrm{v}^{\mathrm{t}}$ as the displayed speed limit of the current horizon, where $\mathrm{M}$ is updated by the following expression:

$$
M=\left\{\begin{array}{c}
M+1, \quad \text { if } v(i)>v^{t} \\
M \quad \text { if } v(i)=v^{t} \\
M-1 \quad \text { if } v(i)<v^{t}
\end{array}\right.
$$

- The new displayed speed limit for the next horizon will readjusted with the predetermined increment $\Delta$, based on the value of $\mathrm{M}$ :

$$
v^{t+1}=\left\{\begin{array}{cc}
v^{t}+\Delta, & \text { if } M>0 \\
v^{t} & \text { if } M=0 \\
v^{t}-\Delta & \text { if } M<0
\end{array}\right.
$$

\subsection{HIGHWAY CAPACITY UNDER CAV IMPACT}

According to existing studies, the application of CAV technologies can improve highway capacity and efficiency. Chen et al. (2017) conducted a comprehensive study to show how CAVs improve highway capacity and efficiency at a macroscopic level. They found that capacity related to CAV penetration rate, platoon size, inter-vehicle spacing, and lane policies and traffic mixed with CAVs and HVs can lead to higher capacity and strict segregation of CVs and HVs will bring lower capacity. Based on the extended analysis, they get the conclusion that CAVs utilize the most efficient lanes on highway, leading them to make the best use of roadway efficiency and gain the highest capacity. On the contrary, Ghiasi et al. (2017) came to the opposite conclusion, based on the analytical stochastic formulation for mixed traffic highway capacity, that higher CAV penetration rates and platooning intensities do not always help improve highway capacity. Therefore, the CAV penetration rate needs to be further analyzed to understand how it can impact highway capacity and efficiency.

For convenience of discussion, this project selects single-lane highways and two-lane highways for study. For a single-lane highway segment, its capacity can be estimated by the inter-vehicle spacing. Many existing studies defined this spacing as the distance between the reference points of an immediate follower and its leader, such as from front bumper to front bumper. The spacing as shown in Figure 3.4, S0 denotes the spacing between HVs; S1 indicates the spacing between $\mathrm{HV}$ and CAV (CAV ahead); S2 denotes the spacing between CAV; and S3 denotes the spacing between CAV and HV (HV ahead). By assuming both CAVs and HVs drive at a constant free 
flow speed and based on the previous study finished by Chen et al. (2017), the relations among all type of spacings can be expressed as $\mathrm{S} 2<\mathrm{S} 0<\mathrm{S} 3<\mathrm{S} 1$. It means the CAV can save intervehicle spacing, which further indicates that $\mathrm{CAV}$ can help improve roadway capacity. When multiple CAVs are bunched, a cooperative adaptive cruise control (CACC) control can be activated and spacing between CAVs will be S2.

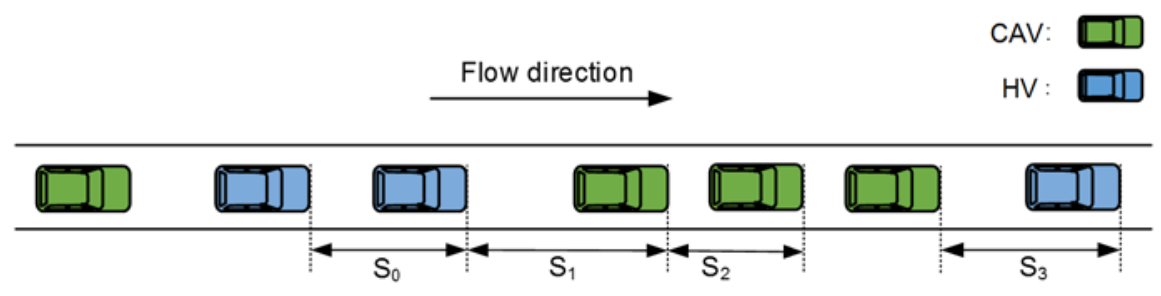

Figure 3.4: Inter-vehicle spacing in traffic mixed with CAVs and HVs

Such a phenomenon can be further proved in the fundamental diagram, as shown in Figure 3.5, where $\mathrm{C}_{0}$ denotes lane capacity with HVs only; $\mathrm{C}_{1}$ denotes lane capacity with HVs and CAVs; $q$ denotes the traffic flow; $u_{0}$ denotes the free-flow speed until reaching critical capacity with HVs only; $u_{1}$ denotes the free-flow speed until reaching critical capacity with RVs and CAVs; $w_{0}$ denotes the free-flow speed until reaching congested with HVs only; $w_{1}$ denotes the free-flow speed until reaching congested with HVs and CAVs; and $d$ denotes the corresponding density under these two different traffic environments. With reduced vehicle spacing, it can be observed in Figure 3.5 that the highway capacity with mixed CAVs and HVs are higher than the case with HVs only.

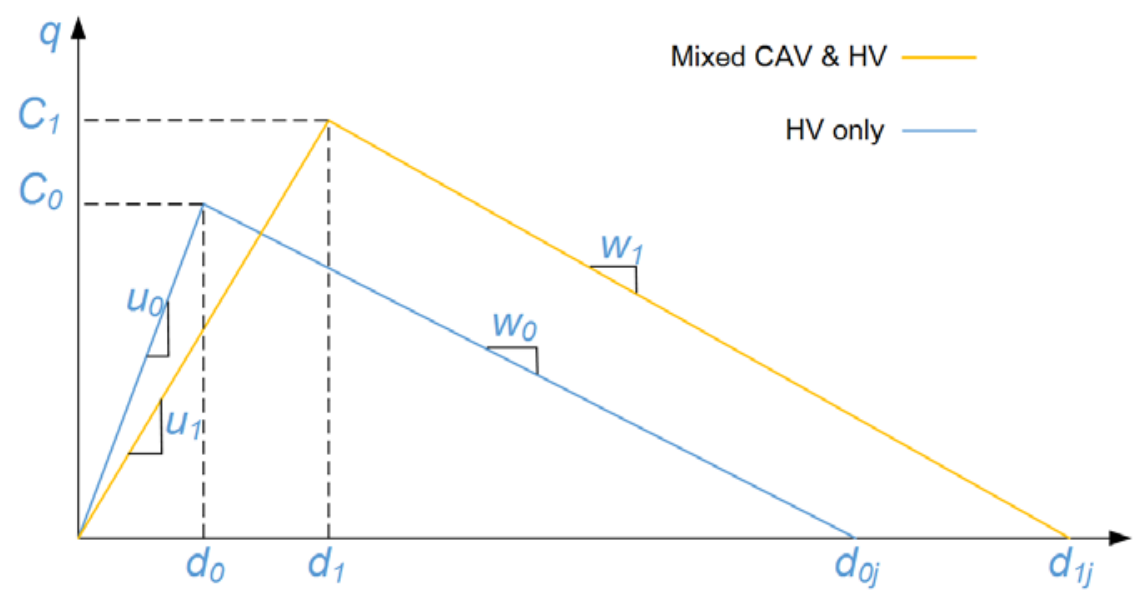

Figure 3.5: Fundamental diagram with HV only and mixed with CAV and HV

For a two-lane highway segment, the capacity estimation under CAV impact becomes more complicated and is subject to many factors. However, on a multilane highway segment, HVs can choose to make lane changes when it follows a slow CAV. Figure 3.6 illustrates a case in which CAVs are traveling at a lower speed compared with HVs. Due to the light traffic condition, HVs can easily pass CAVs by making a few lane-changing maneuvers. Hence, the impact of CV speed control on the entire traffic stream is quite minimal in such cases. In contrast, Figure 3.7 
shows a congested highway segment where HVs have to follow CAVs due to the difficulty of making lane changes. Under such conditions, it can be expected that the speed control of CAVs can greatly affect the entire traffic flow.

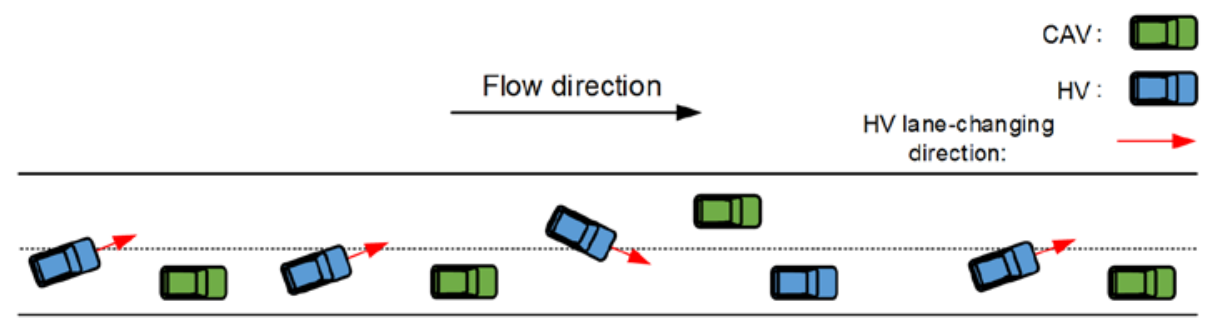

Figure 3.6: Fundamental diagram with HV only and mixed with CAV and HV

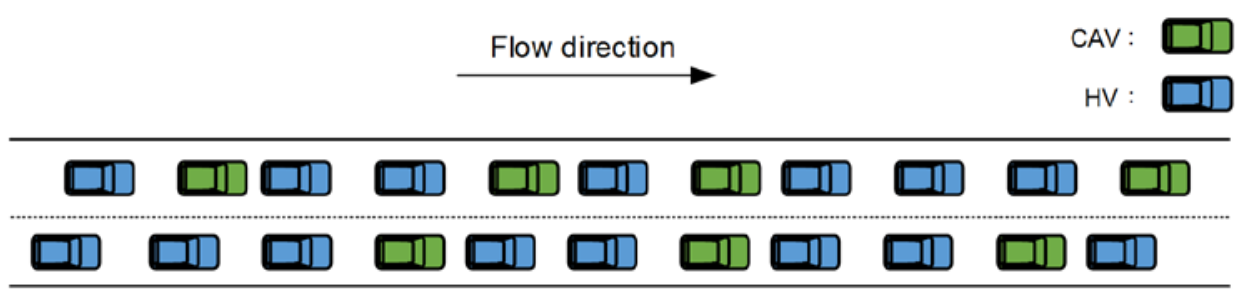

Figure 3.7: Fundamental diagram with HV only and mixed with CAV and HV

From the microscopic level, movement of traffic streams can be characterized by both carfollowing models and lane-changing models. Under the mixed CAV and HV pattern, carfollowing behaviors can be categorized as four categories: CAV follows CAV, CAV follows $\mathrm{HV}, \mathrm{HV}$ follows CAV, and HV follows HV. Similar to the case of a one-lane highway, the main variation of these four following scenarios is the different minimum following spacing. For modeling the discretionary lane-changing behaviors, a probabilistic form has been widely used in existing simulation tools, where the probability is subject to the lane speed variations and number of safe lane-changing gaps. Applying the same logic to analyze the mixed CAV and HV traffic pattern, it can be concluded that more HV lane-changing behaviors may be observed if 1) CAVs are operated at lower speeds; and 2) the number of safe lane-changing gaps is sufficient. Therefore, given the CAV optimal speed control pattern and highway traffic demand profile, there shall exist a critical CAV penetration rate that can maximize the CAV impact to the traffic stream without increasing lane changing significantly.

\subsection{EXPERIMENTAL TESTS}




\subsection{STUDY SITE}

To evaluate the effectiveness of the proposed system in improving freeway operational efficiency, this project selects one freeway segment of I-15 in Salt Lake City as the study site. As shown in Figure. 4.1, the whole stretch is divided into eight segments, where each has a length of 500 meters. Notably, the freeway stretch includes one on-ramp on the third segment and one offramp on the fifth segment. Model parameters, $\tau, v, \kappa$, and $\delta$, are set as $20 \mathrm{~s}, 35 \mathrm{~km}^{2} / \mathrm{h}, 13$ veh/km/lane, and 1.4 , respectively, for the whole stretch. Other parameters $\mathrm{u}_{\mathrm{f}}, \mathrm{d}_{\mathrm{cr}}$, and $a$ are calibrated to be $120 \mathrm{~km} / \mathrm{h}, 33.5 \mathrm{veh} / \mathrm{km}$ and 1.4324 , respectively, based on the field data collected by the Utah Department of Transportation (UDOT). Each segment upstream of Segment 8 has a resulting capacity of 2,000 veh/h/lane, while each segment downstream of Segment 8 (including Segment 8) has a capacity of 1,500 veh/h/lane. In addition, the set of factors $\beta(\mathrm{k})$ which can reflect the impact degree of speed difference between connected vehicles and regular cars can be given 0.5 initially (calibrated by Lu, 2016) based on the specific traffic condition of the numerical example. Because of the existing of CAVs in the mixed flow which are provided with optimal desired speeds, all segments are assumed to have CAVs involved. Figure. 4.2 shows the inflow of Segment 1 and on-ramp, while the off-ramp exiting rate is 12percent.

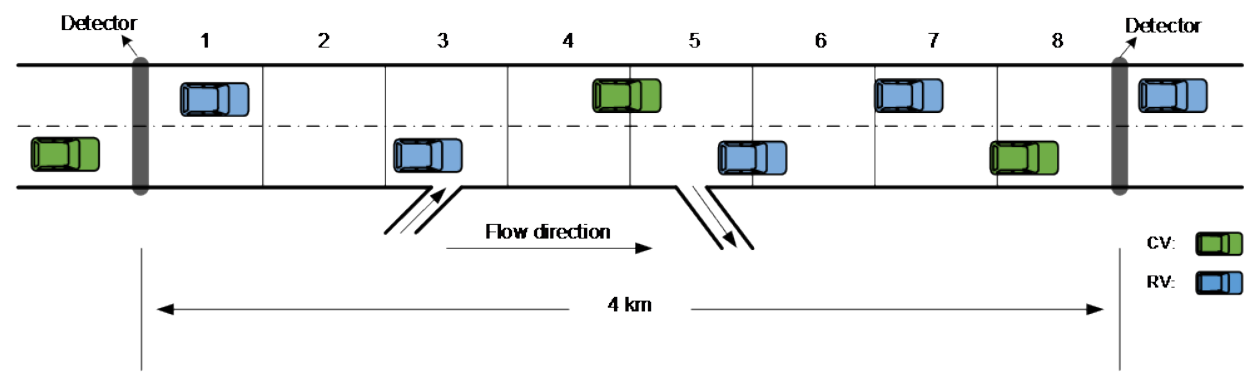

Figure 4.1: Freeway stretch at the study site

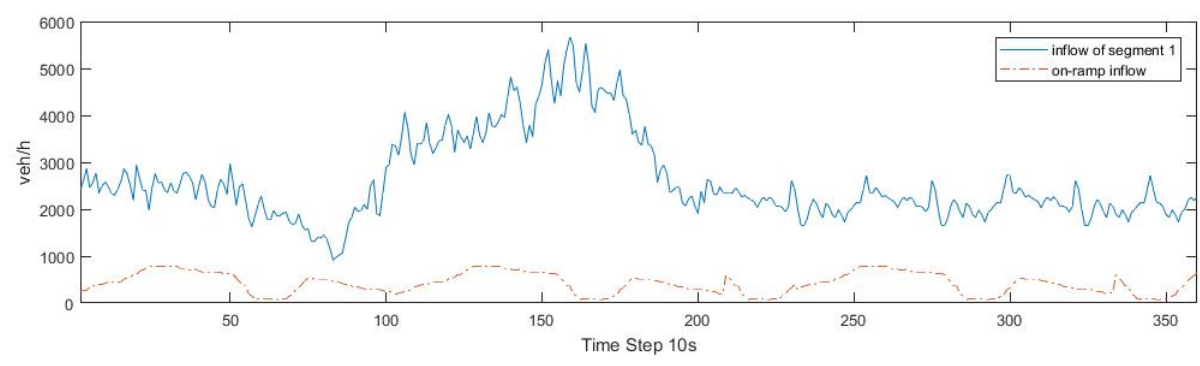

Figure 4.2: The inflows of the study site

\subsection{SIMULATION RESULTS ANALYSIS}

To evaluate the performance of the proposed models, we conducted extensive Monte Carlo simulations grounded on the macroscopic traffic flow model. Firstly, based on the objective of minimizing the total travel time, the distribution of mean speeds on each freeway segment under 
different percentages of CAVs in mixed flow (20percent, 50percent, 70percent and 100percent) are shown in Figure. 4.3-Figure. 4.6. Based on the simulation results, one can observe that the time period (i.e., 100-250 time step) is identified to be peak period. The concurrent congestion was formed at Segment 8 and was propagated back to Segment 1. Comparing the distributions of mean speed under No-CAVs control and With-CAVs control, one can reflect that the WithCAVs control with different percentages of connected vehicles in mixed flow all can strongly offer a higher speed during high traffic demand periods. In addition, increasing the penetration rate of CAVs can help improve the system's operational efficiency.
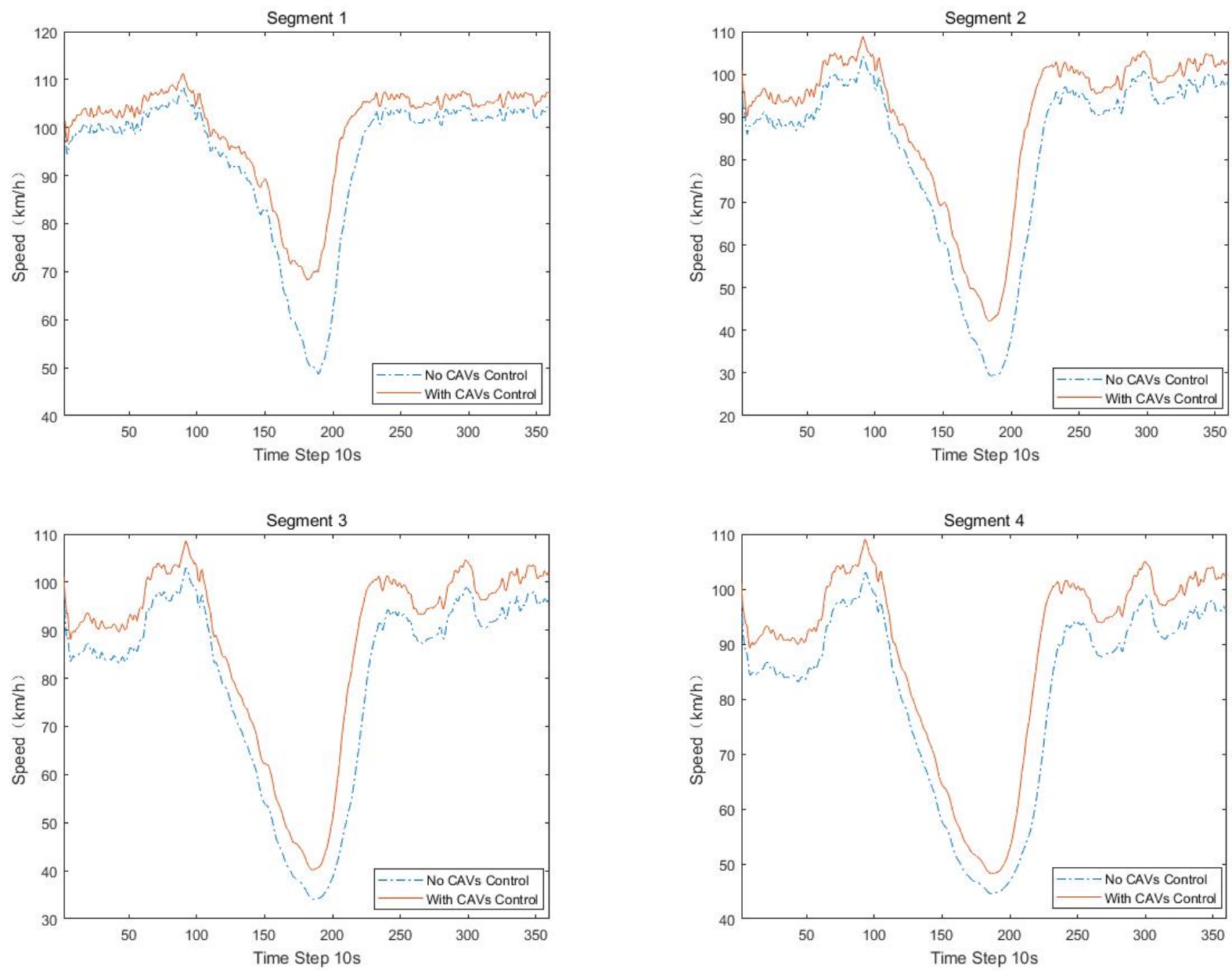

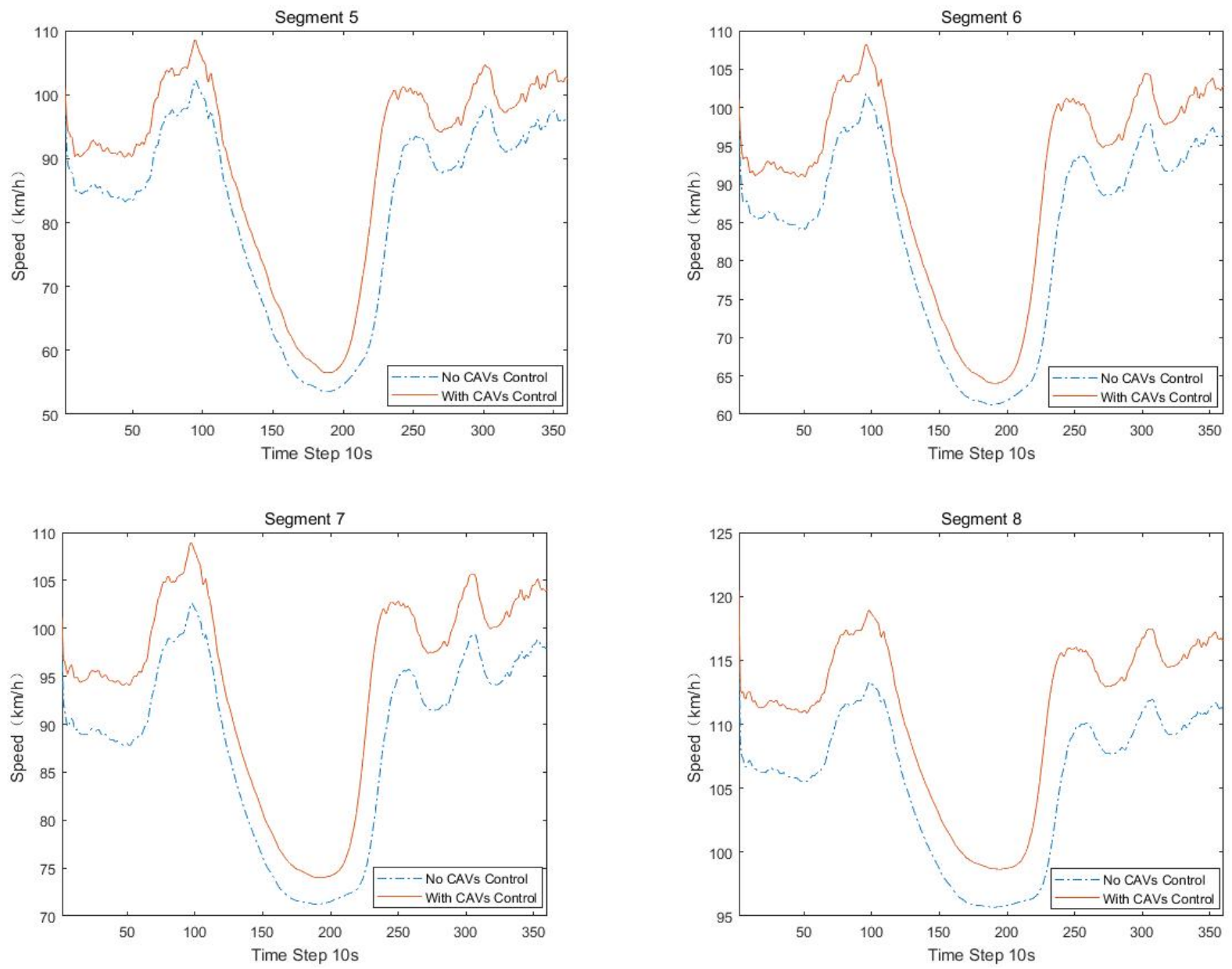

Figure 4.3: Mean speed with 20percent of connected vehicles in mixed flow
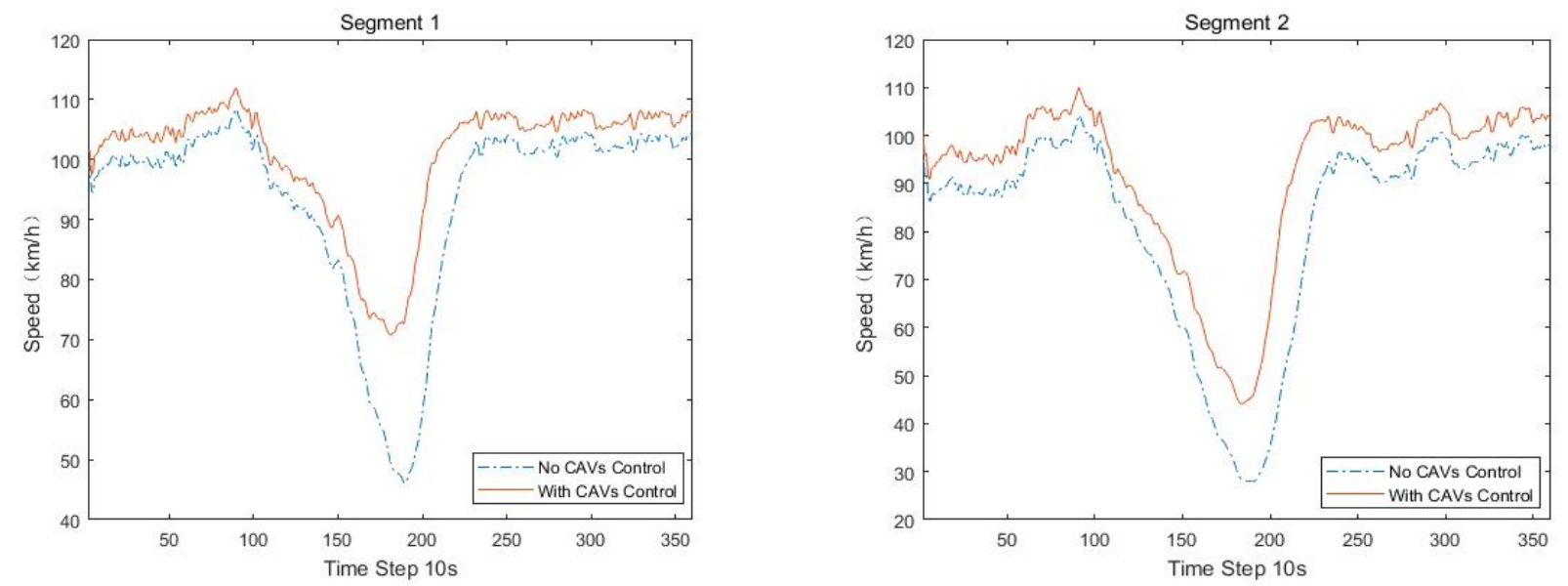

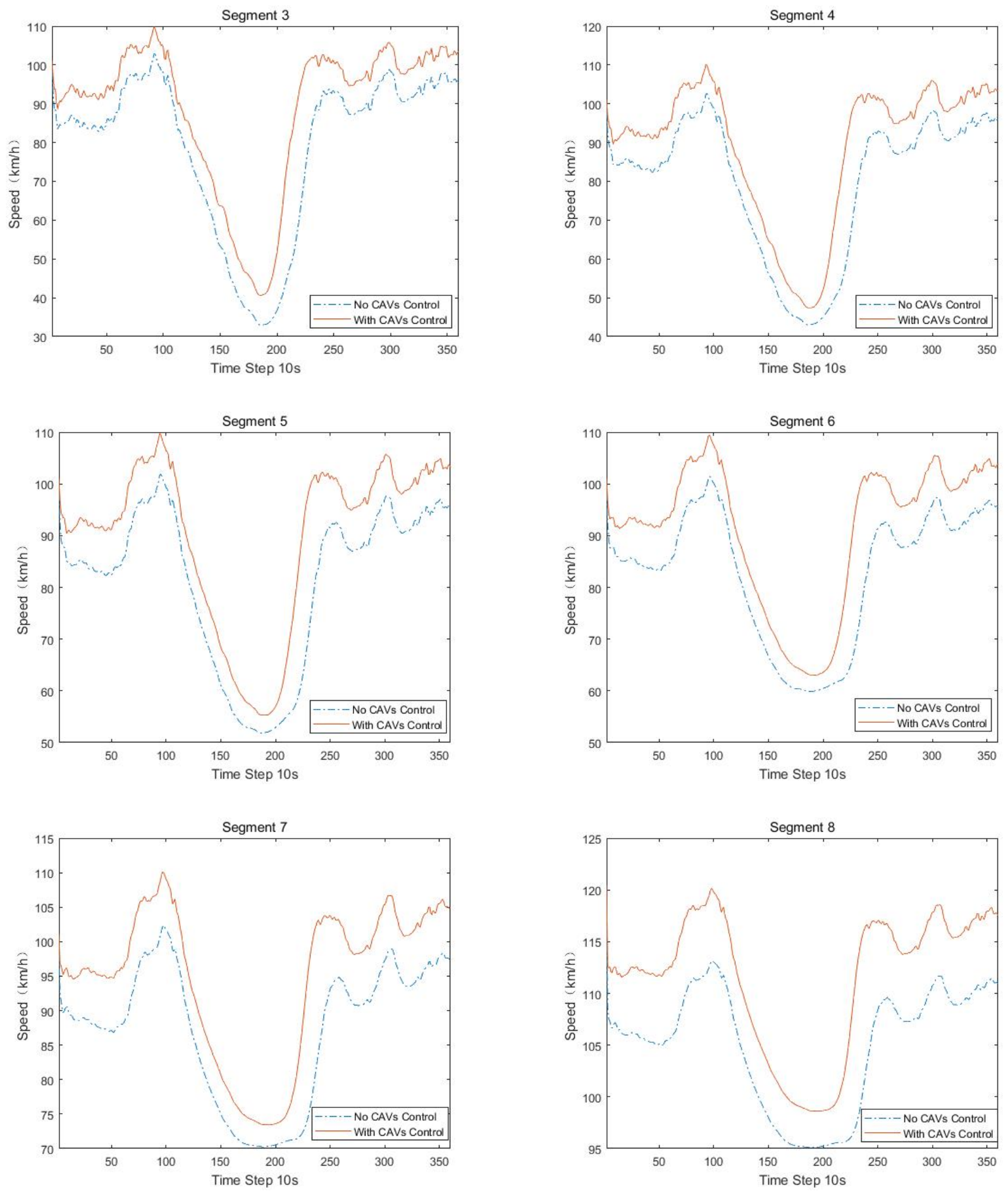

Figure 4.4: Mean speed with 50percent of connected vehicles in mixed flow 

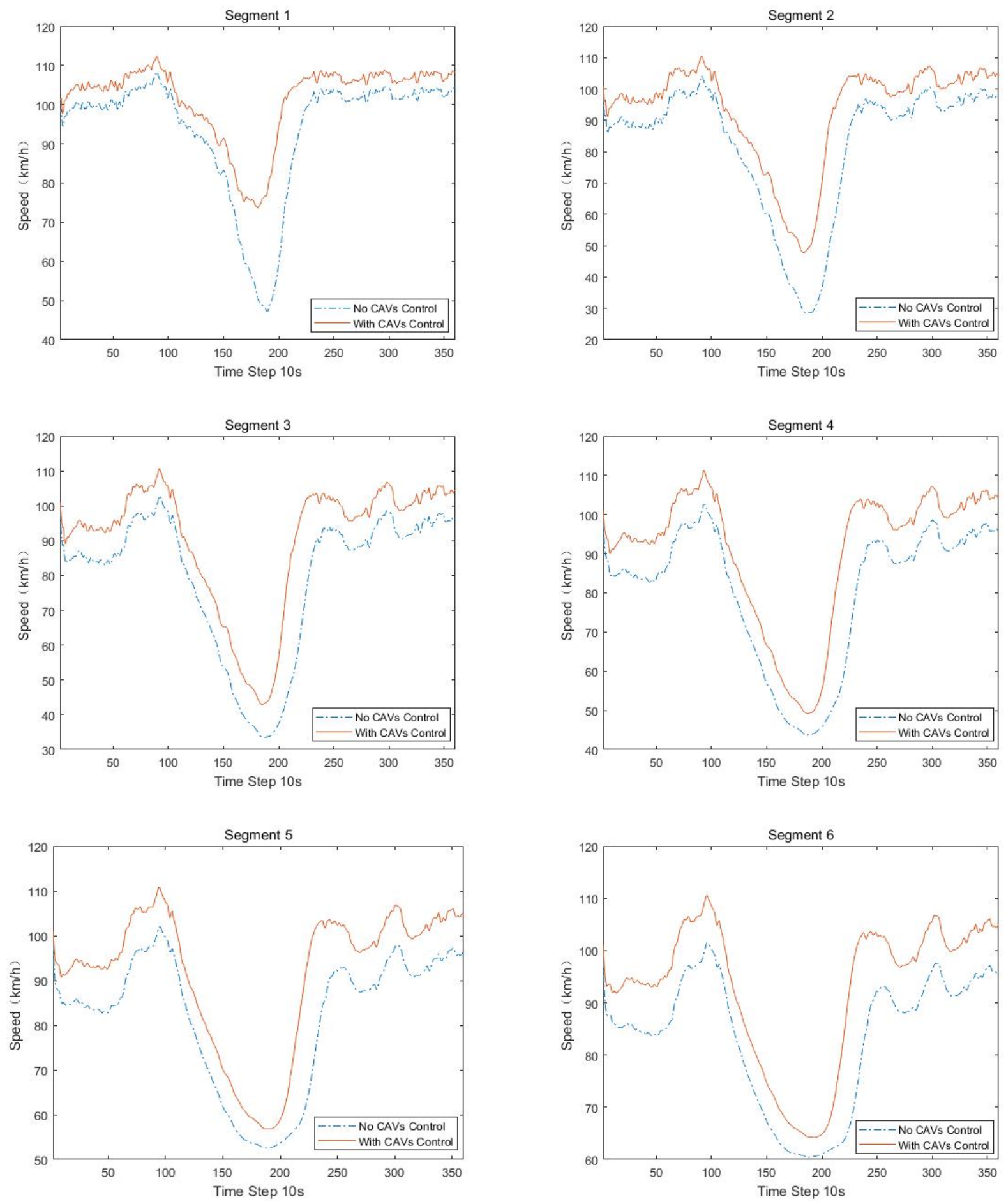

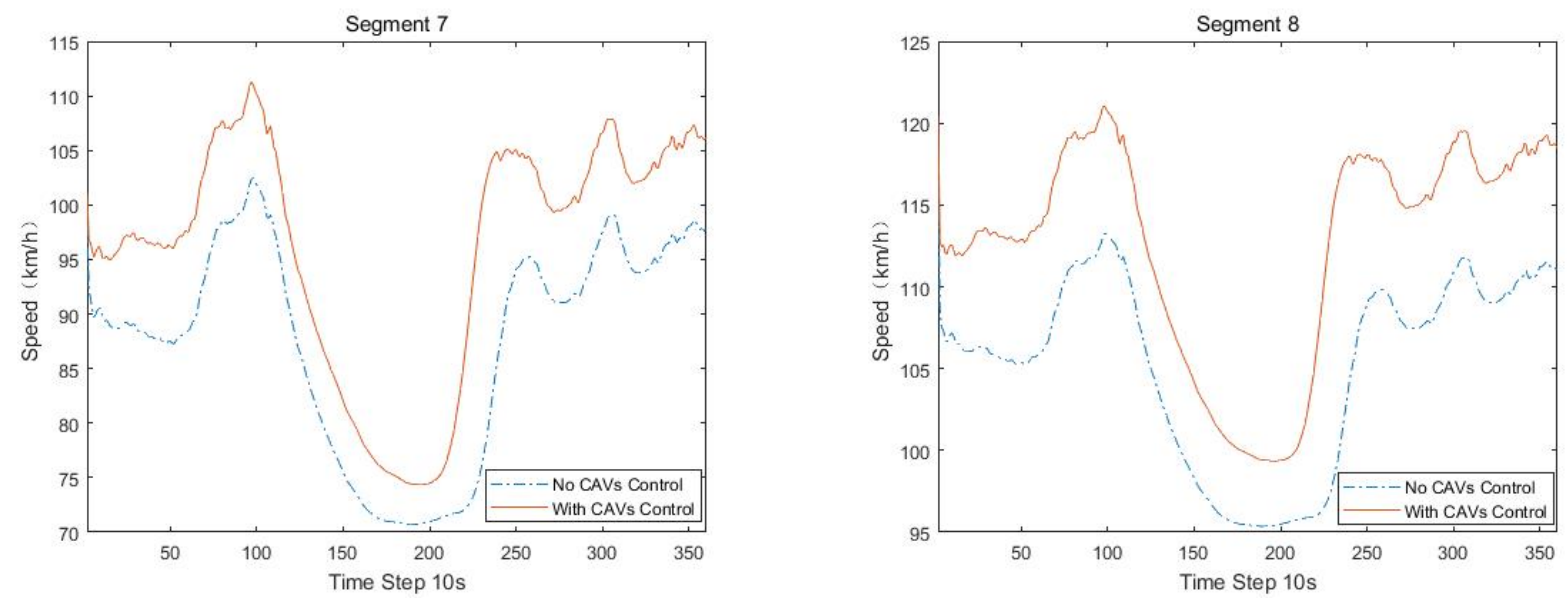

Figure 4.5: Mean speed with 70percent of connected vehicles in mixed flow
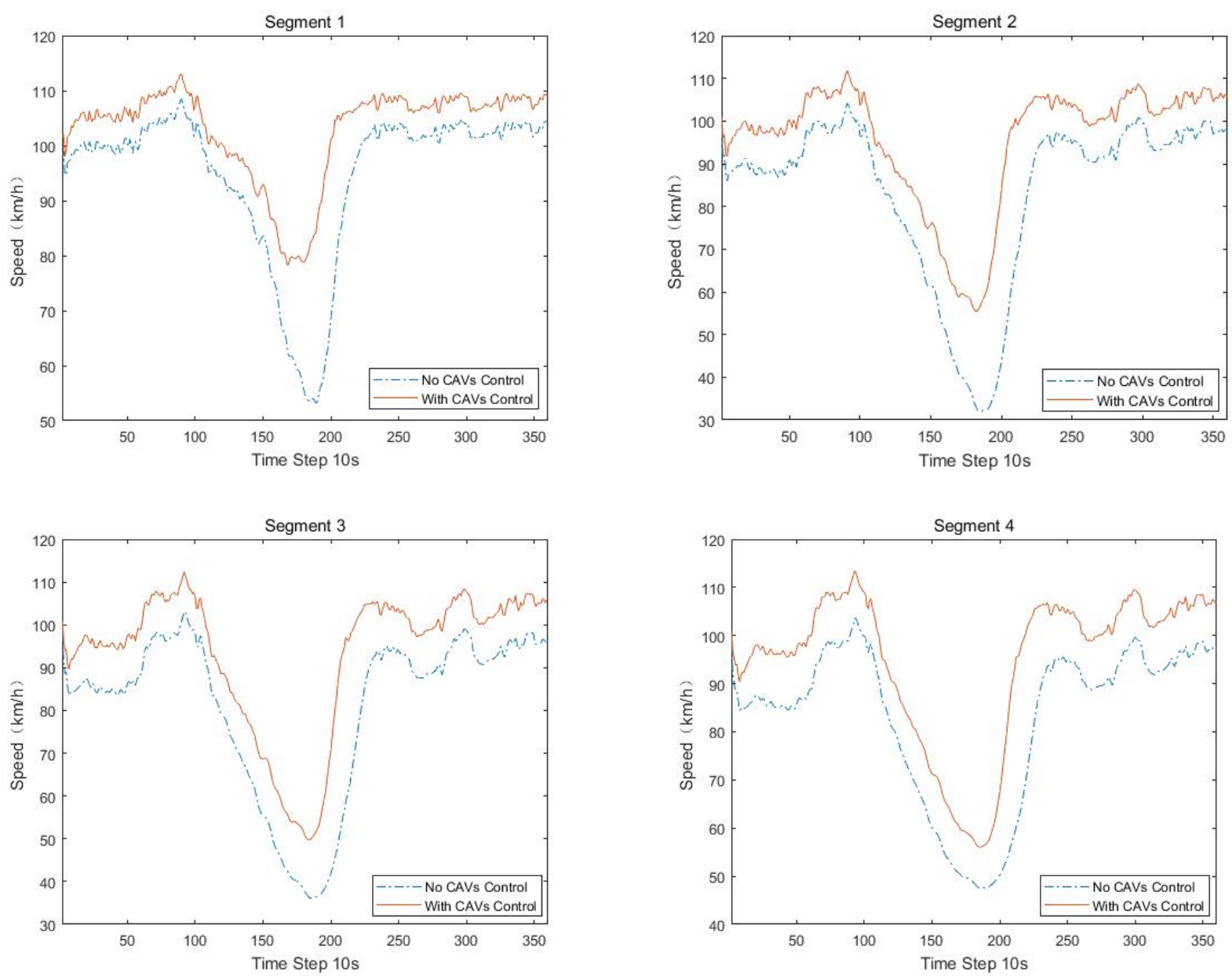

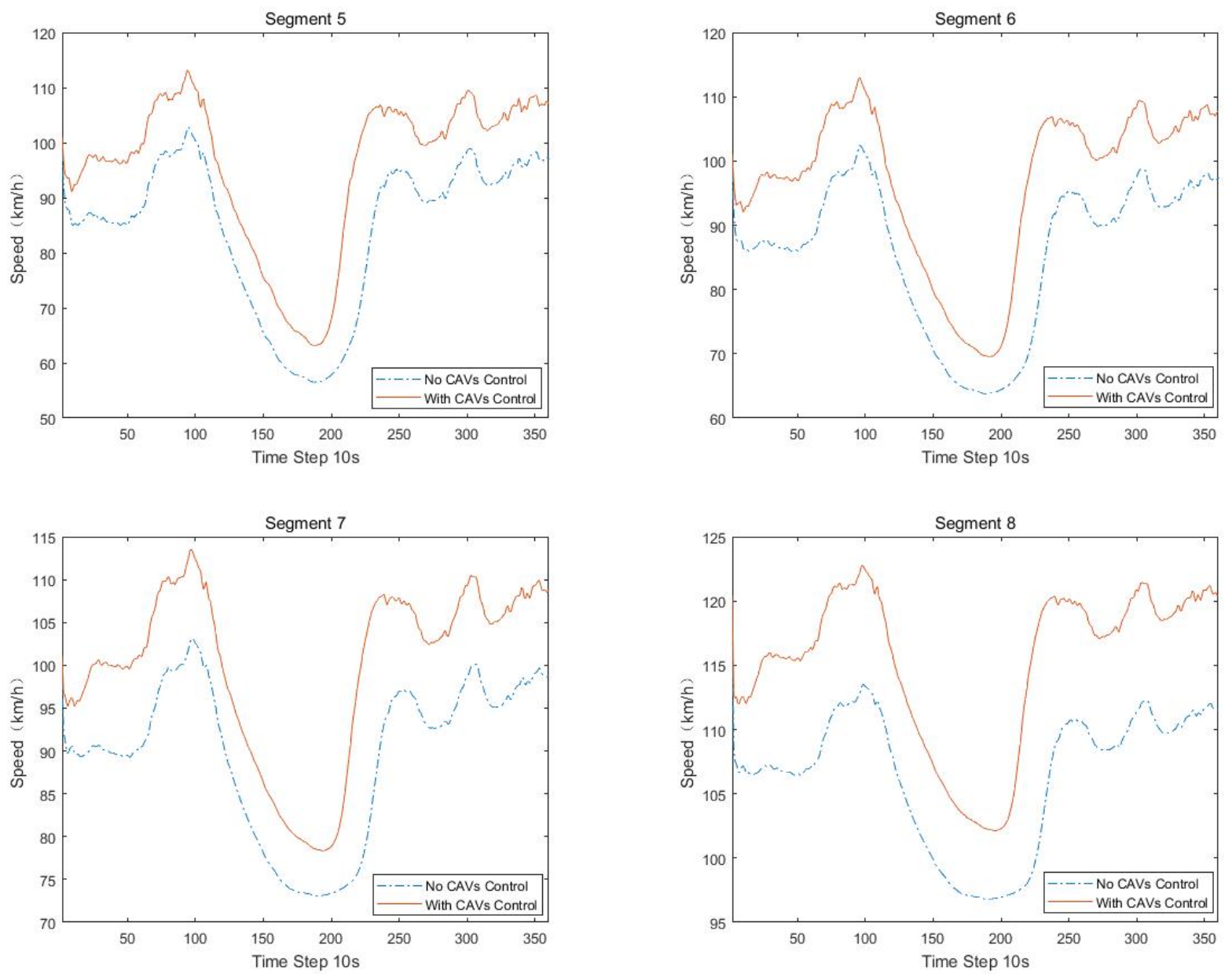

Figure 4.6: Mean speed with 100percent of connected vehicles in mixed flow

To further evaluate traffic control efficiency more effectively, the time-dependent travel time is one of the most important measure of effectiveness (MOE). As the time-dependent travel time is directly affected by the time-dependent density on each segment, Figure. 4.7 to Figure. 4.10 show the resulting density under the No-CAVs control and With-CAVs control models with the objective of minimizing travel time under different percentages of CAVs in mixed flow. Notably, the density starts to increase when the freeway has high traffic demands which were determined by the inflows. Compared with the No-CAVs control, the density is reduced under the With-CAVs control. 

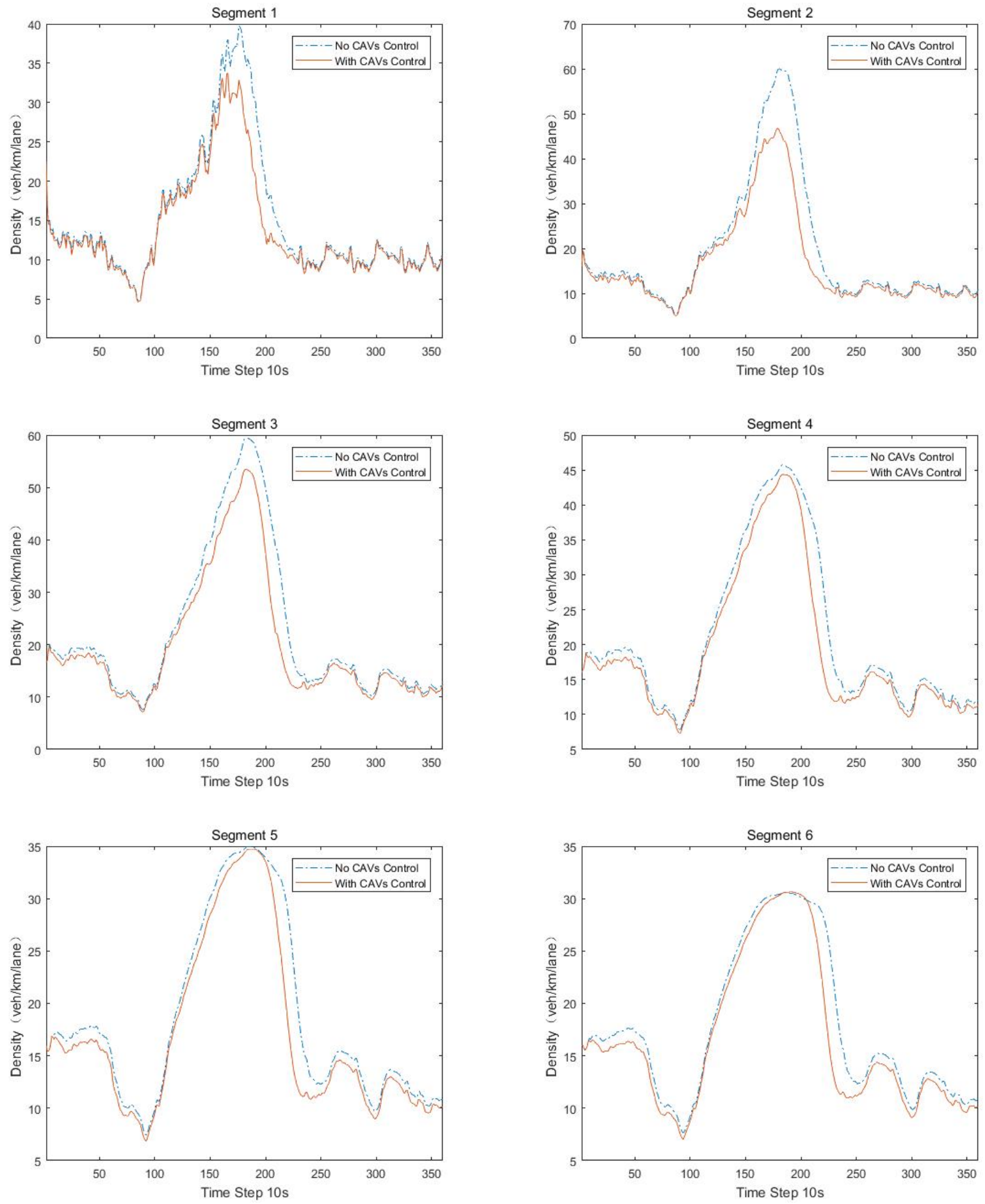

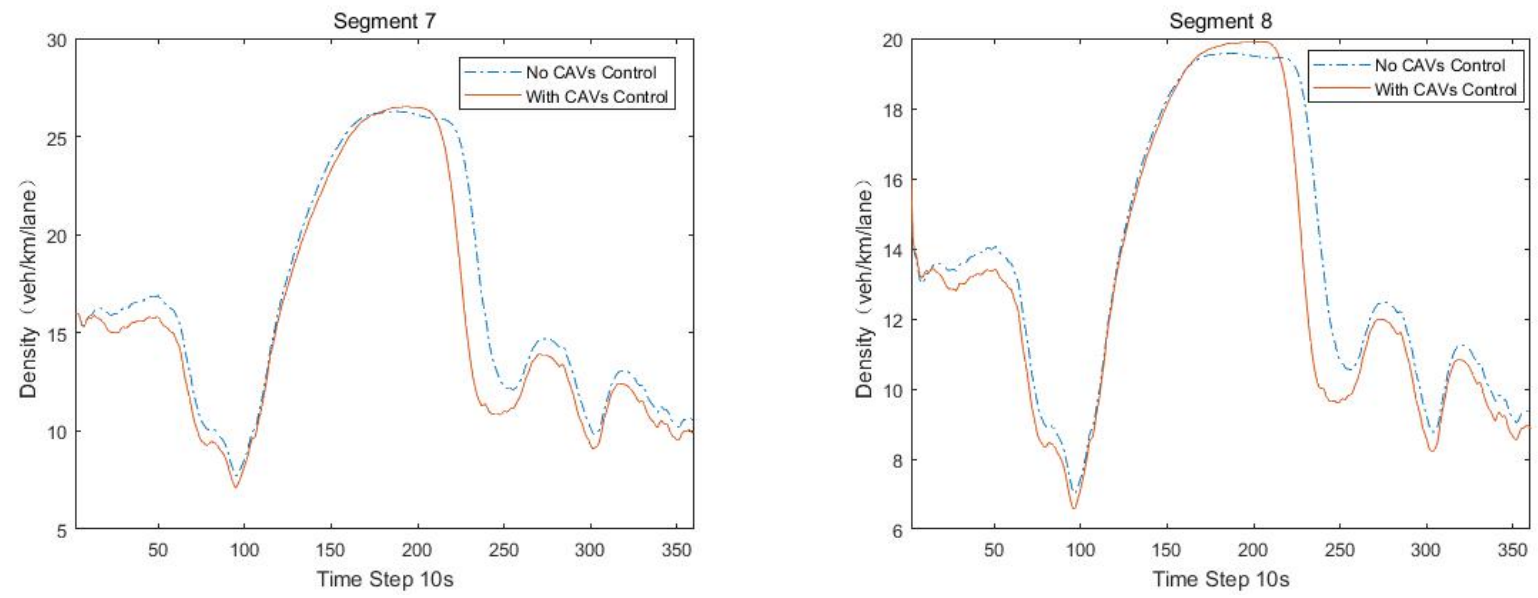

Figure 4.7: Density with 20percent of connected vehicles in mixed flow
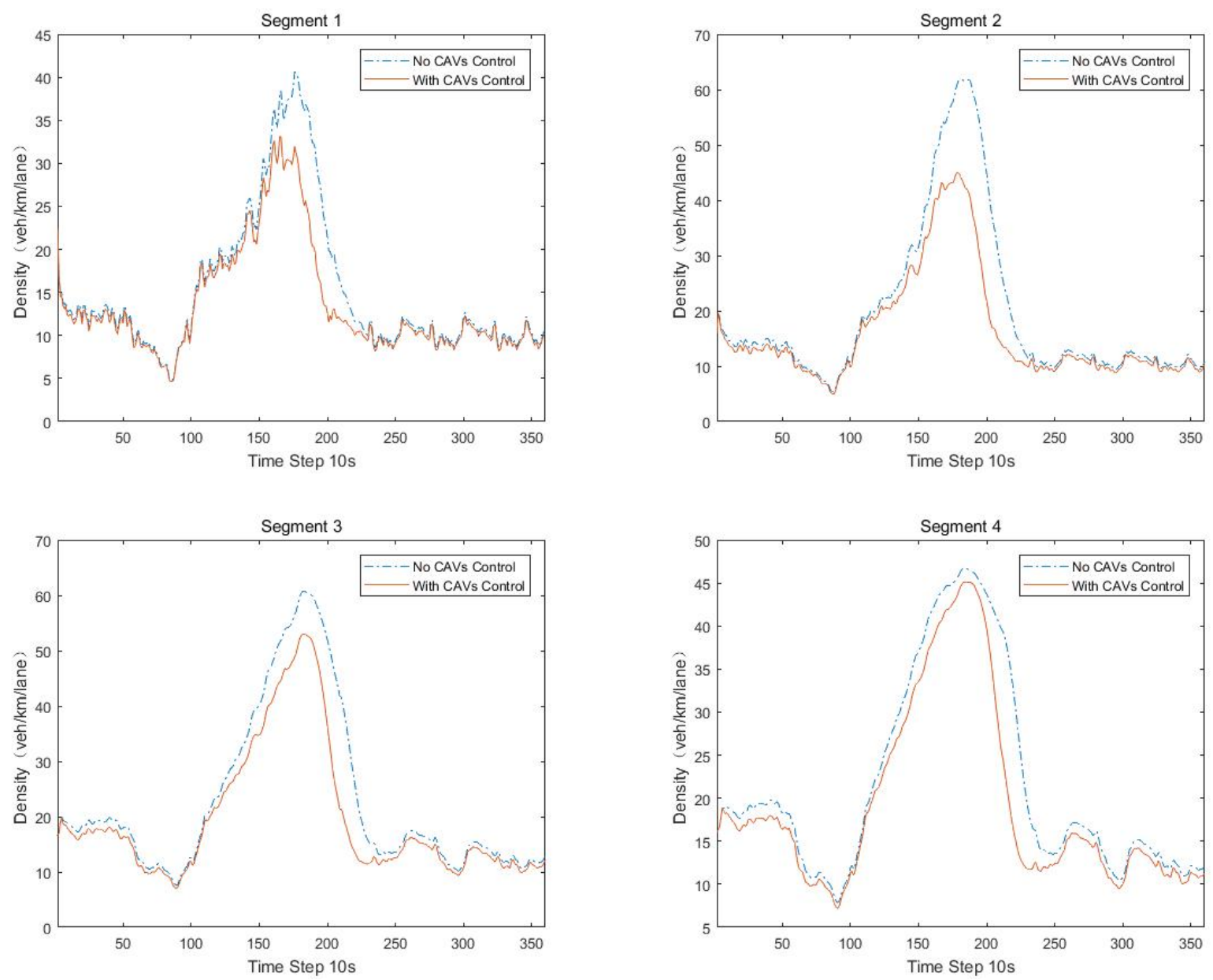

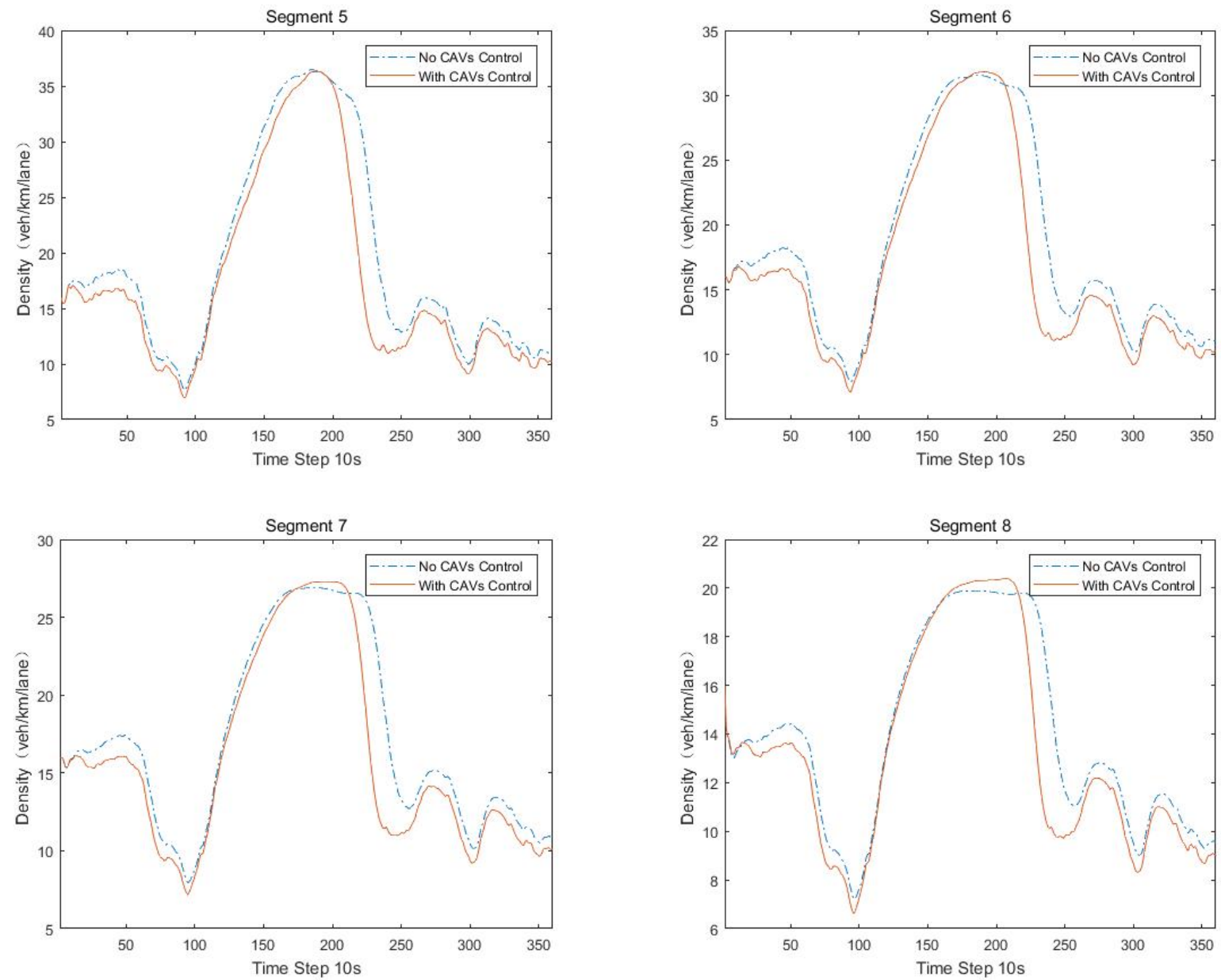

Figure 4.8: Density with 50percent of connected vehicles in mixed flow
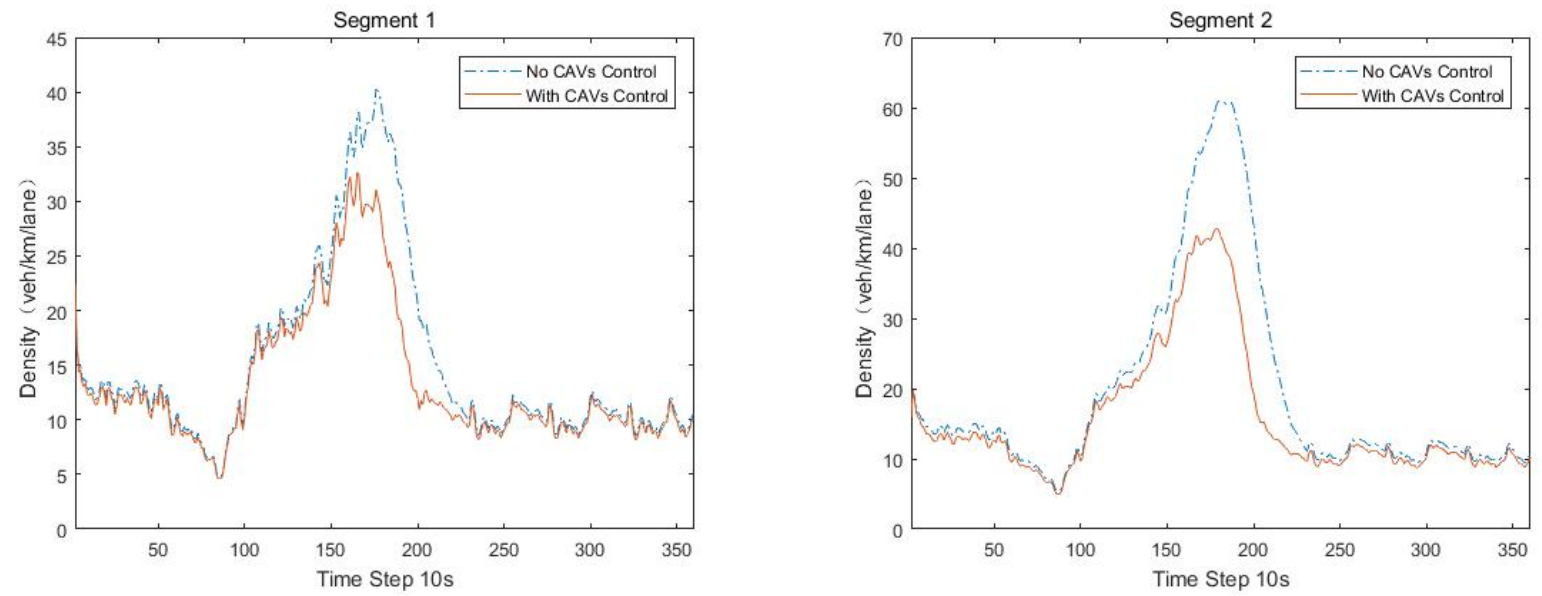

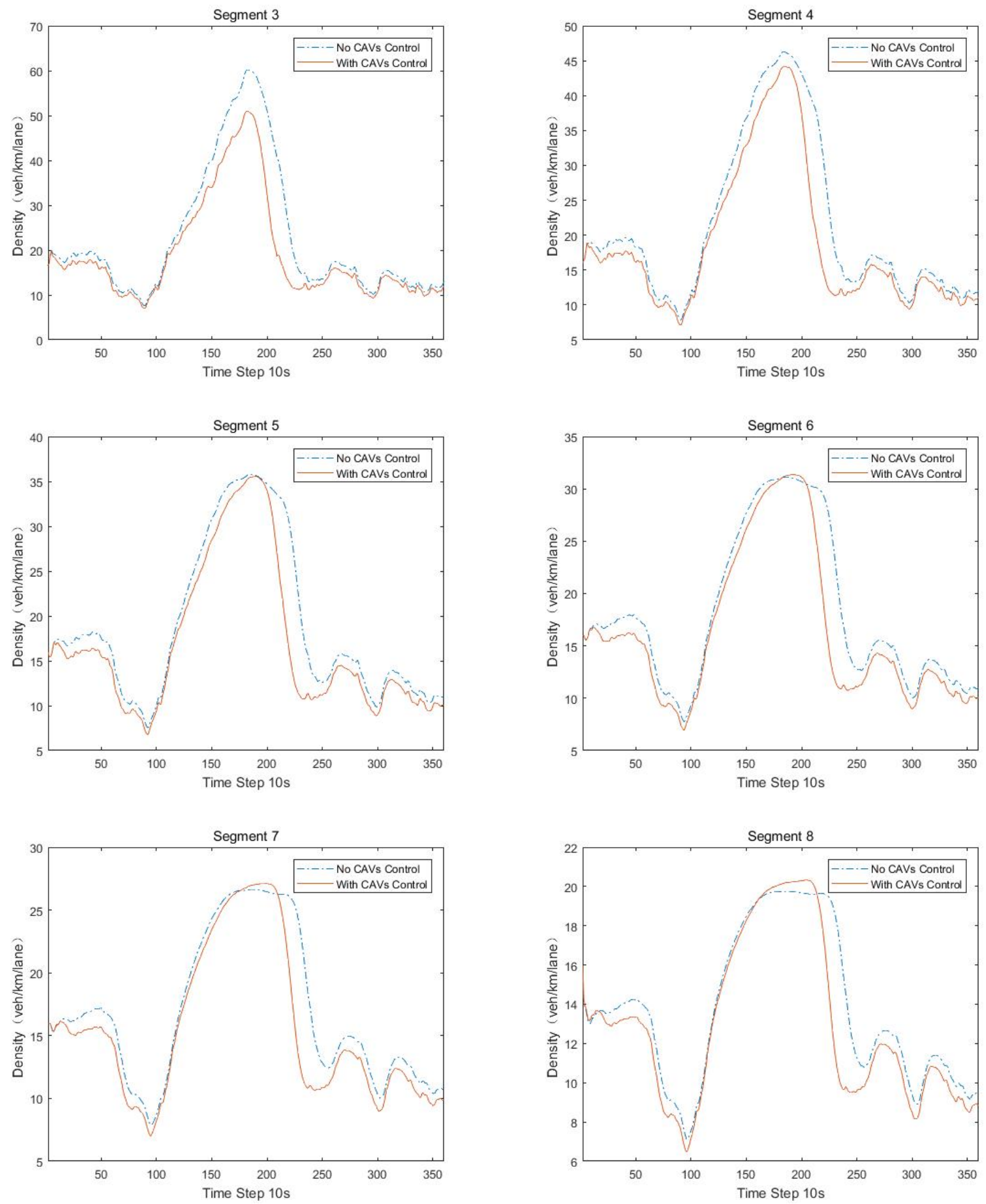

Figure 4.9: Density with 70percent of connected vehicles in mixed flow 

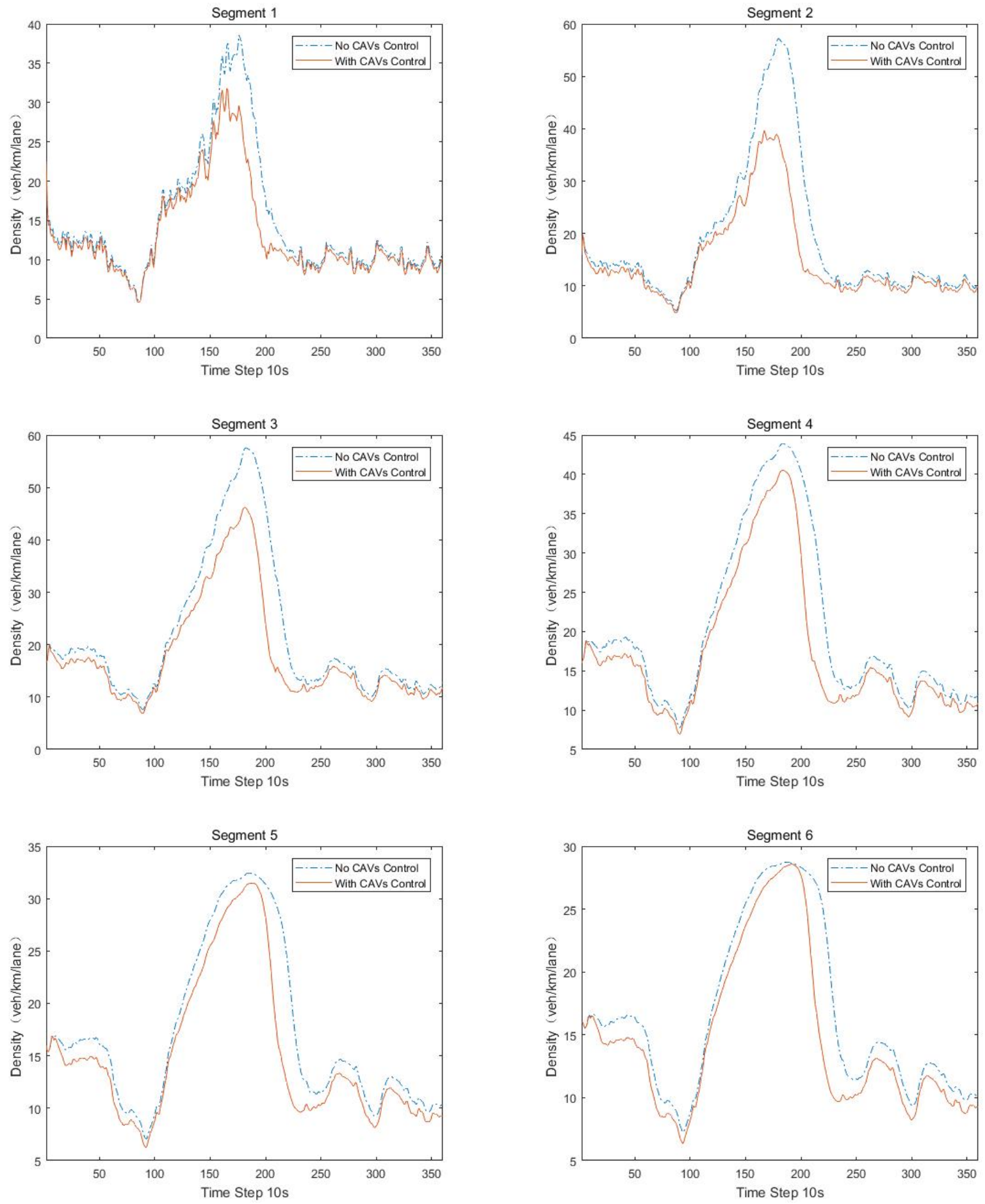

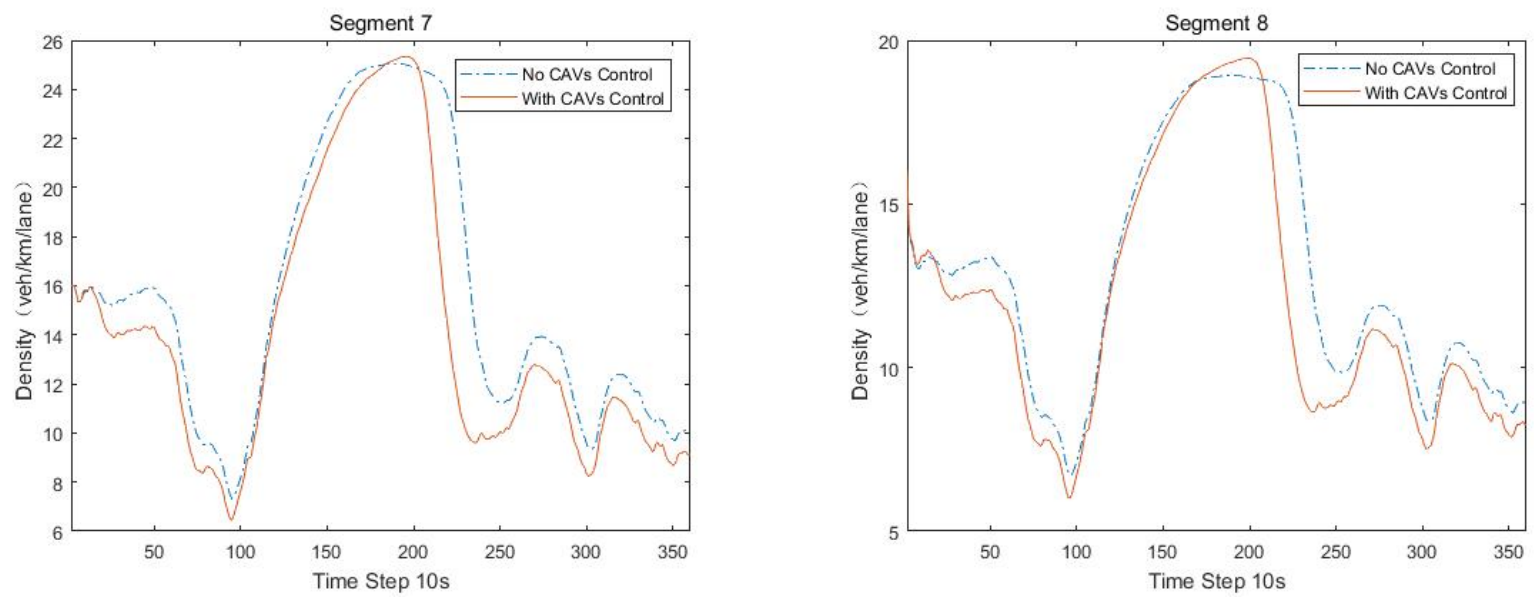

Figure 4.10: Density with 100percent of connected vehicles in mixed flow

To compare the performance of CAVs speed control with different percentages of CAV in mixed flow, the average delay is selected as another MOE to reflect the improvement in traffic conditions. The comparisons are shown by Table 4.1 and Table 4.2. In these tables, one can observe that a higher ratio of CAVs in mixed will yield more improvement in delay time reductions. Hence, higher percentages of CAV in mixed flow will have more improvement in traffic efficiency with the objective of minimizing total travel time .

Table 4.1: Average delay during time periods with different ratio of connected vehicles in mixed flow

\begin{tabular}{l|l|l|l|l|l|l}
\hline Ave Delay & $\mathbf{1 - 1 0}(\mathbf{m i n})$ & $\mathbf{1 1 - 2 0}(\mathbf{m i n})$ & $\mathbf{2 1 - 3 0} \mathbf{( m i n})$ & $\mathbf{3 1 - 4 0} \mathbf{( m i n})$ & $\mathbf{4 1 - 5 0}(\mathbf{m i n})$ & $\mathbf{5 1 - 6 0}(\mathbf{m i n})$ \\
\hline No CAVs & $38.40 \mathrm{sec}$ & $26.72 \mathrm{sec}$ & $92.74 \mathrm{sec}$ & $94.11 \mathrm{sec}$ & $29.61 \mathrm{sec}$ & $26.33 \mathrm{sec}$ \\
\hline $20 \%$ CAVs & $29.95 \mathrm{sec}$ & $19.61 \mathrm{sec}$ & $77.84 \mathrm{sec}$ & $71.50 \mathrm{sec}$ & $21.96 \mathrm{sec}$ & $19.25 \mathrm{sec}$ \\
\hline $50 \%$ CAVS & $28.77 \mathrm{sec}$ & $18.25 \mathrm{sec}$ & $76.32 \mathrm{sec}$ & $70.45 \mathrm{sec}$ & $20.56 \mathrm{sec}$ & $17.88 \mathrm{sec}$ \\
\hline $70 \%$ CAVs & $27.35 \mathrm{sec}$ & $16.91 \mathrm{sec}$ & $71.89 \mathrm{sec}$ & $62.77 \mathrm{sec}$ & $19.09 \mathrm{sec}$ & $16.55 \mathrm{sec}$ \\
\hline $100 \%$ CAVs & $24.36 \mathrm{sec}$ & $14.28 \mathrm{sec}$ & $61.32 \mathrm{sec}$ & $45.81 \mathrm{sec}$ & $16.15 \mathrm{sec}$ & $13.94 \mathrm{sec}$ \\
\hline
\end{tabular}

Table 4.2: Percentage improvement on average delay comparing CAVs controls with no CAVs controls

\begin{tabular}{l|c|c|c|c|c|c}
\hline Ave Delay & $\mathbf{1 - 1 0}(\mathbf{m i n})$ & $\mathbf{1 1 - 2 0}(\mathbf{m i n})$ & $\mathbf{2 1 - 3 0}(\mathbf{m i n})$ & $\mathbf{3 1 - 4 0}(\mathbf{m i n})$ & $\mathbf{4 1 - 5 0}(\mathbf{m i n})$ & $\mathbf{5 1 - 6 0}(\mathbf{m i n})$ \\
\hline No CAVs & $/$ & $/$ & $/$ & $/$ & $/$ & $/$ \\
\hline $20 \%$ CAVs & $22.01 \%$ & $26.61 \%$ & $16.07 \%$ & $24.03 \%$ & $25.84 \%$ & $26.89 \%$ \\
\hline $50 \%$ CAVS & $25.08 \%$ & $31.70 \%$ & $17.71 \%$ & $25.14 \%$ & $30.56 \%$ & $32.09 \%$ \\
\hline $70 \%$ CAVs & $28.78 \%$ & $36.71 \%$ & $22.48 \%$ & $33.30 \%$ & $35.53 \%$ & $37.14 \%$ \\
\hline $100 \%$ CAVs & $36.56 \%$ & $46.56 \%$ & $33.88 \%$ & $51.32 \%$ & $45.46 \%$ & $47.06 \%$ \\
\hline
\end{tabular}




\subsection{ANALYSIS CAV IMPACT TO HIGHWAY CAPACITY}

To further study the CAV impact on the HVs, this study has simulated the mixed traffic flow under various traffic patterns, as shown in Table 4.3.

Table 4.3: Mixed traffic flow simulation scenarios

\begin{tabular}{l|l|l}
\hline Demand Level (two lanes) & CV Penetration Rate & CV Flows (two lanes) \\
\hline $1,500 \mathrm{veh} / \mathrm{hr}$ & $5 \%-80 \%$ with 5\% increment & $75-1,200 \mathrm{veh} / \mathrm{hr}$ \\
\hline $2,000 \mathrm{veh} / \mathrm{hr}$ & $5 \%-80 \%$ with $5 \%$ increment & $100-1,600 \mathrm{veh} / \mathrm{hr}$ \\
\hline $2,500 \mathrm{veh} / \mathrm{hr}$ & $5 \%-80 \%$ with $5 \%$ increment & $125-2,000 \mathrm{veh} / \mathrm{hr}$ \\
\hline $3,000 \mathrm{veh} / \mathrm{hr}$ & $5 \%-80 \%$ with $5 \%$ increment & $150-2,400 \mathrm{veh} / \mathrm{hr}$ \\
\hline $3,500 \mathrm{veh} / \mathrm{hr}$ & $5 \%-80 \%$ with 5\% increment & $175-2,800 \mathrm{veh} / \mathrm{hr}$ \\
\hline $4,000 \mathrm{veh} / \mathrm{hr}$ & $5 \%-80 \%$ with 5\% increment & $200-3,200 \mathrm{veh} / \mathrm{hr}$ \\
\hline
\end{tabular}

In the control model, CAV speed impact on the HV group is represented by the key factor $\beta(k)$. Notably, the value of the factors shall be subject to both demand levels and CV ratios. Following the similar procedure of an existing study (Yang, 2016), $\beta(k)$ is calibrated with microscopic simulations and the corresponding value within different simulation scenarios are shown in Figure 4.11.

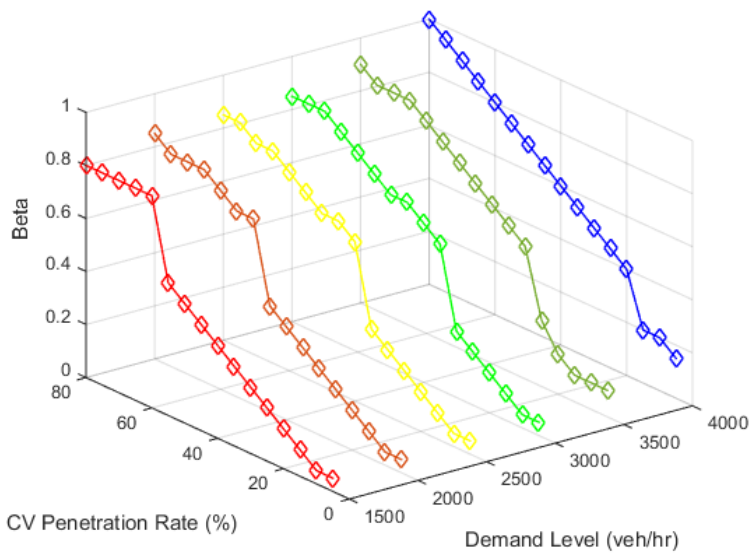

Figure 4.11: The distribution of $\beta \mathrm{i}$ in different scenarios

Selecting average travel time of all vehicles along studied freeway segments as the performance index, this study has simulated all scenarios designed in Table 4.3 and the results are summarized in Figure 4.12. By comparing the cases of different CAV penetration rates, it can be observed that the average travel time will be reduced with the increase of the CV penetration rate, which prove the benefit of implementing CAV technology. Notably, at the low demand level, such a benefit is not significant. For example, with the $1,500 \mathrm{veh} / \mathrm{hr}$ traffic demand, the corresponding travel times of 5percent and 80percent CAV ratios are obtained as 129.1 seconds and 121.4 seconds, respectively. In contrast, in the cases with 4,000 veh/hr upstream arriving flow, the travel time difference by these two CAV ratios is 18 seconds. Hence, in a more traffic congested environment, the optimal CAV speed control system can yield more benefits on reducing vehicle delays. However, it shall be noted that all scenarios are simulated at 
undersaturated conditions. When traffic demand exceeds freeway capacity, the operational benefits of CAVs may be minimized.

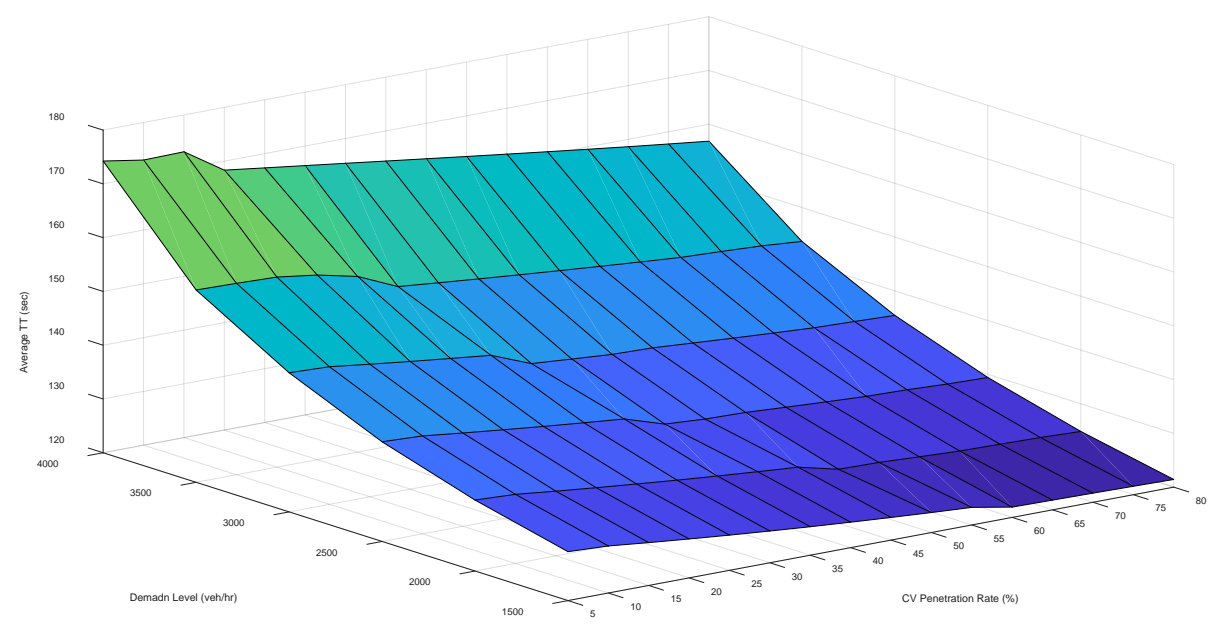

Figure 4.12: Freeway average travel time under different scenarios

To study the CAV impact on HVs and understand their interactions, this research also compared the travel time performance of four different vehicle groups: 1) all vehicles without CV involved; 2) all vehicles with CAV optimal control; 3) CAVs under optimal speed control; and 4) HVs under CAV optimal control. Figure 8 presents the resulting average travel time under different CAV rates and demand levels. By comparing the cases under CAV control with the ones without CAVs, it can be noted that providing advisory speeds to CAVs can help reduce travel time of the entire traffic stream. Also, CAVs experienced much lower travel time compared with HVs in all scenarios. This is due to the capability of optimal speed control on mitigating shock wave impacts caused by bottleneck locations. With the increase of the CAV penetration rate, the reduction of CAV travel time is quite minimal under low demand conditions. With higher upstream traffic flow, the optimal CAV speed harmonization will yield more travel time reduction when the CAV ratio is increased. In addition, further performance comparison between the CAV and HV groups revealed that the CAV impact, indicated by $\mathrm{HV}$ travel time reduction along with CAV optimal control, becomes more significant when the system involves a larger portion of CAVs. Under each level of demand, there exists a critical CAV penetration rate that can greatly reduce the speed difference between CAVs and HVs. Based on Figure 4.13 (a) (f), the critical CAV rates under various demand patterns and corresponding average travel time of different vehicle groups are summarized in Table 4.3.

Table 4.3: The simulation results of the scenarios with critical CAV ratio

\begin{tabular}{ccccccc}
\hline Demand level & $\mathbf{1 5 0 0}$ & $\mathbf{2 0 0 0}$ & $\mathbf{2 5 0 0}$ & $\mathbf{3 0 0 0}$ & $\mathbf{3 5 0 0}$ & $\mathbf{4 0 0 0}$ \\
\hline Critical CAV Ratio & $60 \%$ & $50 \%$ & $40 \%$ & $35 \%$ & $30 \%$ & $20 \%$ \\
All traffic TT with no CAV & 130.08 & 134.20 & 139.60 & 147.01 & 157.92 & 179.17 \\
All traffic TT with CAV & 121.92 & 126.27 & 131.98 & 139.14 & 149.37 & 168.34 \\
TT of CAV & 121.28 & 125.05 & 129.96 & 136.55 & 146.06 & 163.27 \\
TT of HV & 122.86 & 127.49 & 133.36 & 140.59 & 150.85 & 169.72 \\
TT Difference between CAV and HV & $1.3 \%$ & $2.4 \%$ & $3.4 \%$ & $4 \%$ & $4.7 \%$ & $6.4 \%$ \\
\hline
\end{tabular}




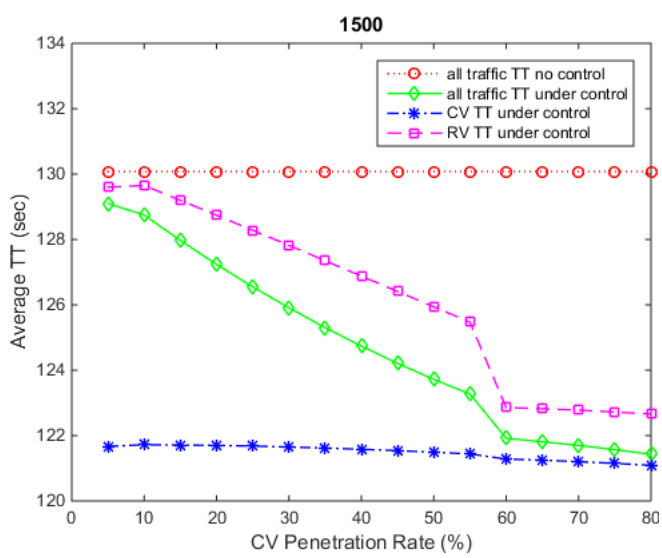

(a)

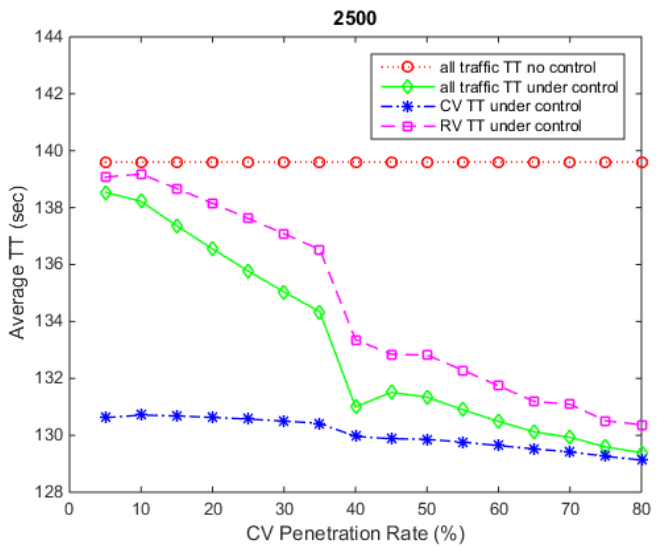

(c)

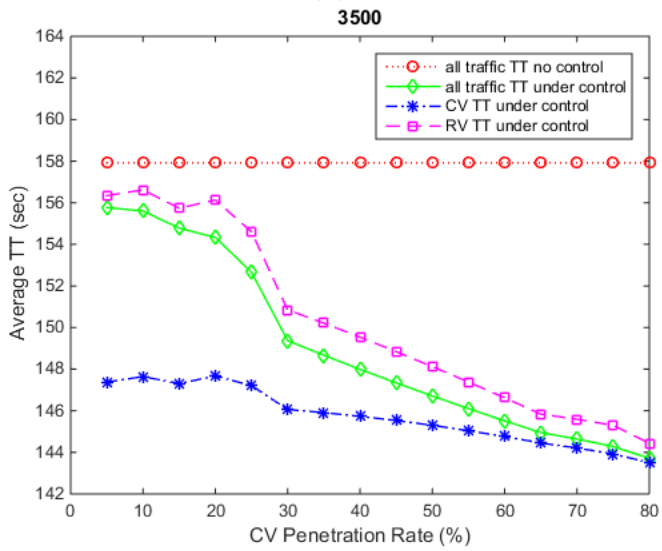

(e)

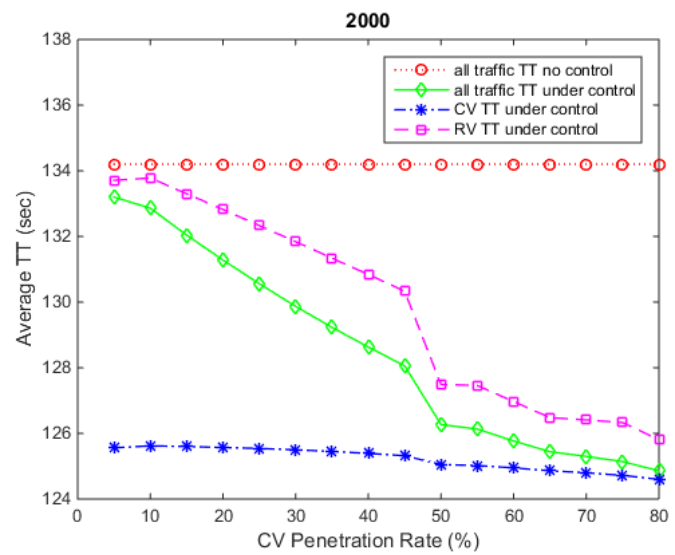

(b)

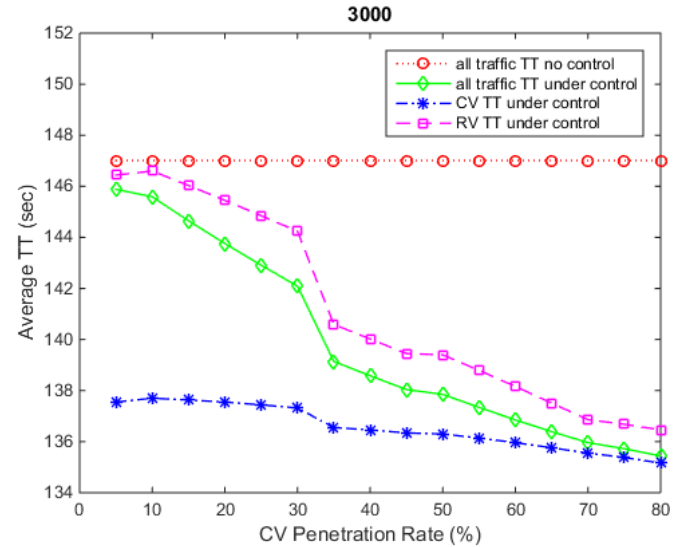

(d)

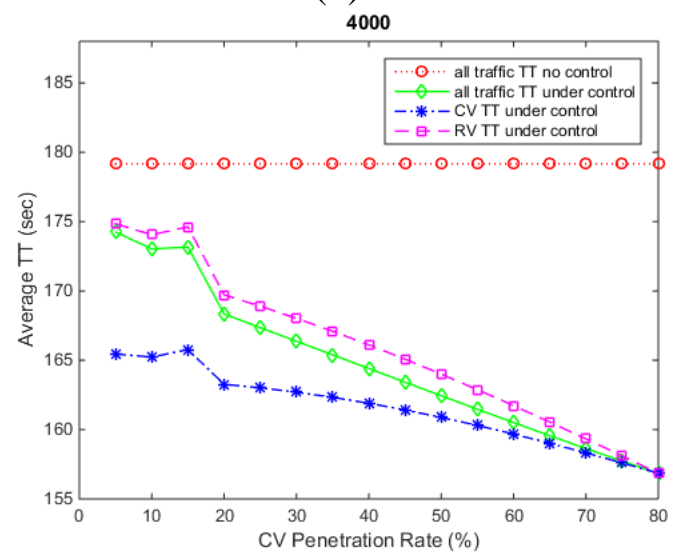

(f)

Figure 4.13: Average travel time comparison between different vehicle groups

To better illustrate the existence of a critical CAV penetration rate and demonstrate how it can impact highway performance, Figure 4.14 summarizes the reduced average travel time of all vehicles under different scenarios, compared with the cases of no CAV control. Notably, under each level of demand, the travel time reduction suddenly becomes significant after the CAV ratio reaches the critical value. After passing the critical CV rates, the average travel time will keep reducing with the increase in CAV rates. However, under the low demand scenarios, such travel time reduction is not significant. 


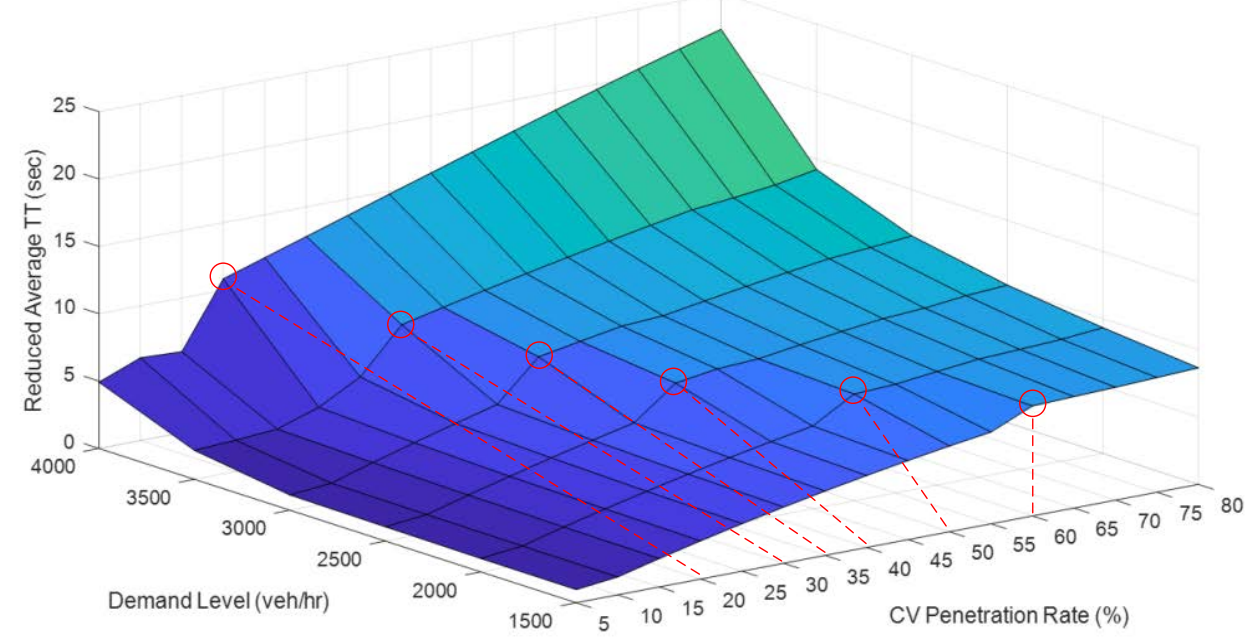

Figure 4.14: Reduced average travel time under different scenarios and critical CV rates

To further analyze the CAV speed impact on HVs under critical CAV rate conditions, Figure 10 presents the time-dependent travel time of all traffic, CAVs and HVs. In each case, it is shown that CAV optimal control can help reduce the travel time of both CAVs and HVs. Also, HVs have very similar travel time compared with CAVs. Hence, one can conclude that HVs were very likely to follow CAVs within the traffic stream when the CAV rate reached a critical value under each level of demand.

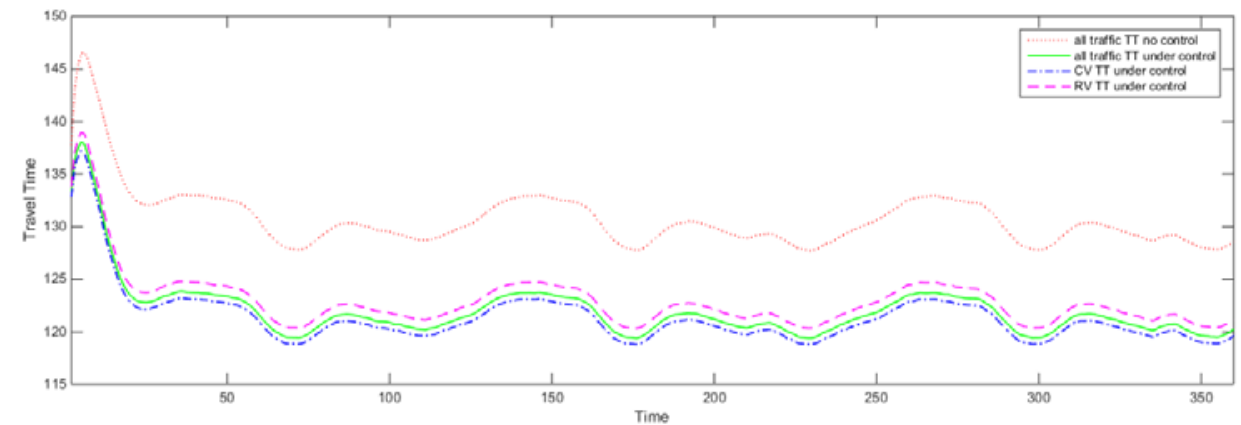

(a) level of demand: $1,500 \mathrm{veh} / \mathrm{hr}$

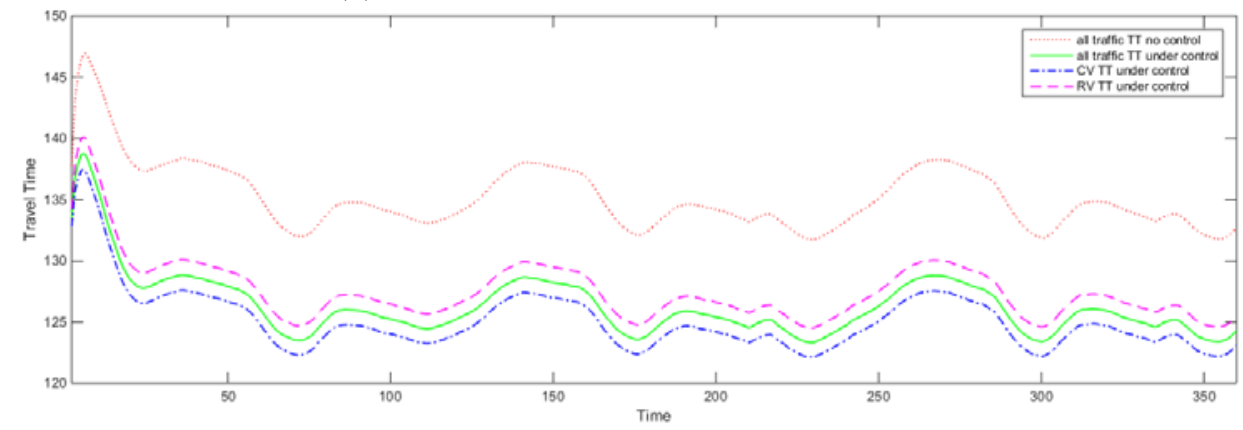

(b) level of demand: 2,000 veh/hr 


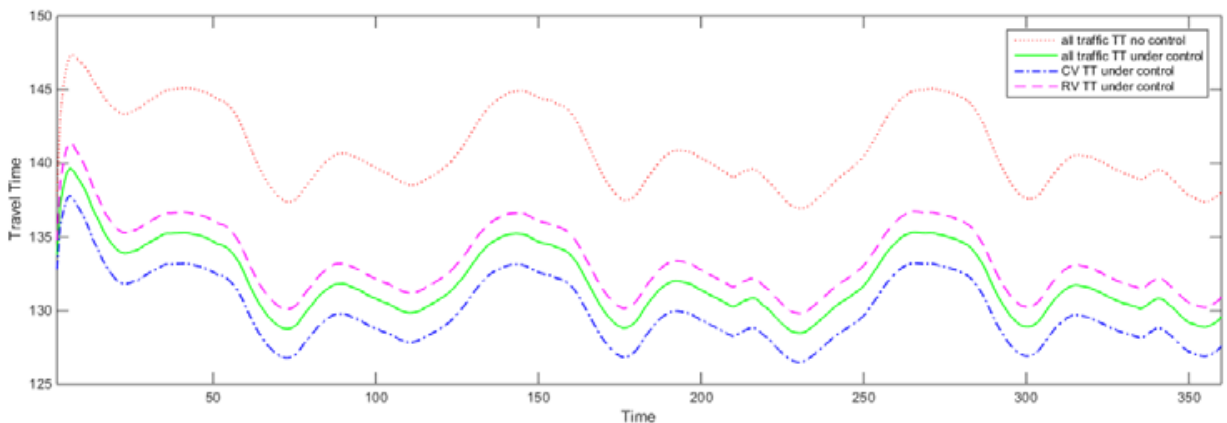

(c) level of demand: 2,500 veh/hr

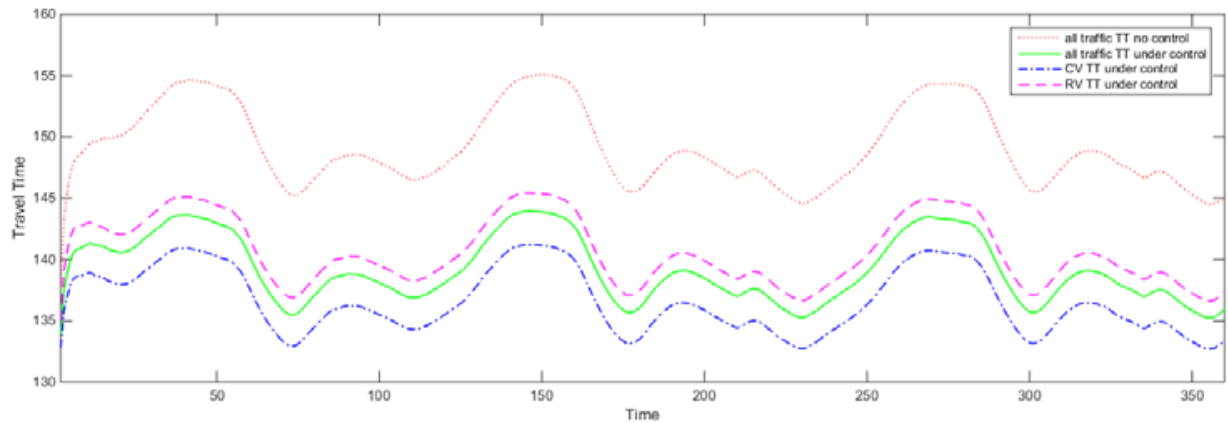

(d) level of demand: 3,000 veh/hr

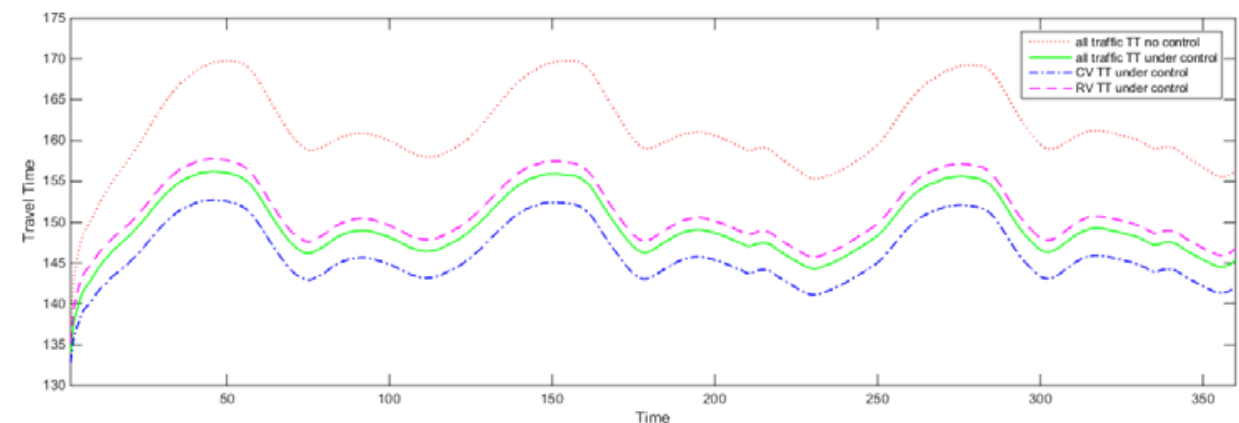

(e) level of demand: 3,500 veh/hr

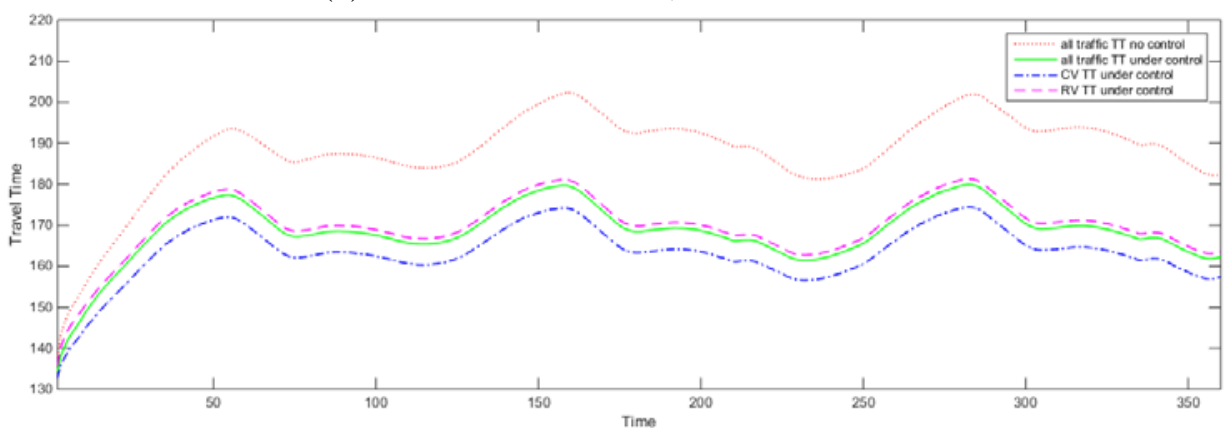

(f) level of demand: 4,000 veh/hr

Figure 4.15: Time-dependent travel time under different levels of demand

In summary, we can summarize the following key findings based on the comprehensive simulation analysis;

- within the mixed CAV and HV traffic pattern, performing optimal speed control to CAVs will concurrently benefit HVs since they are sharing the roadway; 
- given a demand pattern on a target freeway segment, there should exist a critical CAV penetration rate that can greatly improve the performance of CAV-based control systems;

- once the CAV penetration rate reached the critical value, the speed difference between HVs and CAVs will become insignificant; and

- mixed traffic under high demand level (but still below the capacity) intends to have a smaller critical CAV rate compared with the case under low demand level.

Notably, although this study has utilized numerical tests to show the existence of a critical CAV penetration rate and indicated its value was subject to demand levels, it is essential to complete theoretical proof in future studies. Also, more simulation and field studies shall be conducted to provide guidance on how to determine the critical CAV ratio so as to maximize highway capacity.

\subsection{CONCLUSIONS}

\subsection{KEY FINDINGS}

In summary, this project mainly focused on developing a control framework to produce an optimal desired speed profile for CAVs when operating on the freeways. The proposed optimization model was based on a novel traffic state estimation model that can explicitly show the speed interactions between CAVs and HVs. Specifically, when an optimal desired speed is placed on a CAV, it may affect the speed of nearby HVs as they are sharing the road.

Considering that a conventional macroscopic traffic flow model would fall short of capturing such situations, this project implemented an extended model that treats CAVs and HVs as separate vehicle classes and their interdependency of speeds is represented by a set of impact factors. Using simulations to evaluate the proposed system, results analysis revealed that the proposed models can effectively reduce freeway travel time of both CAVs and HVs, compared with the no-CAV cases. Further sensitivity analysis on CAV penetration rates also indicated that improving the CAV penetration rate would benefit the reduction of traffic delays. The proposed models can serve as the foundation of many other CAV applications on freeways, such as Cooperative Adaptive Cruise Control (CACC).

This project also studied the CAV impact on highway operational performance under a mixed CAV and HV environment. According to the different traffic characteristics between CAVs and HVs, our research firstly analyzed how implementation of CAVs can affect highway capacity under both one-lane and multilane cases. Then a hypothesis was made that there shall exist a critical CAV penetration rate that can maximize the benefits of CAV implementations. To prove this concept with numerical analysis, this study implemented the macroscopic traffic flow model along with an embedded CAV speed optimization function to simulate the mixed traffic pattern under various conditions. The simulation results revealed that performing optimal speed control 
to CAVs will concurrently benefit HVs due to their interactions in the traffic streams. The highway capacity will consequently be increased. Also, given a demand pattern on a target freeway segment, there should exist a critical CAV penetration rate that can greatly reduce the speed difference between HVs and CAVs. With the increasing of traffic demand volumes, such a critical CAV rate intends to be smaller on the same freeway stretch

\subsection{FUTURE RESEARCH DIRECTIONS}

Future research directions include: 1) develop theoretical methods to model the CAV impact within the traffic stream; 2) prove the existence of a critical CAV penetration rate that can maximize the CAV implementation benefits; and 3) produce either close-formed equations or guidelines to estimate highway capacity under a mixed CAV and HV environment. 


\subsection{REFERENCES}

Abdel-Aty M, Cunningham RJ, Gayah VV, \& Hsia L (2008). Dynamic variable speed limit strategies for real-time crash risk reduction on freeways. Transportation Research Record; 2078, 108-116.

Ahn, K., Rakha, H., \& Park, S. (2013). Ecodrive application: Algorithmic development and preliminary testing. Transportation Research Record: Journal of the Transportation Research Board, (2341), 1-11.

Andersen, C., (2017). Stranger in a Strange Lane - Automated Vehicles on Human Centric Roads., AAAI workshop: Artificial Intelligence for Connected Automated Vehicles. San Francisco, CA.

Anund A, Ahlström C, Almqvist S, \& Yahya MR (2009). Evaluation of local ITS solutions in urban areas. VTI Report.

Bai, F., Stancil, D. D., \& Krishnan, H. (2010). Toward understanding characteristics of dedicated short range communications (DSRC) from a perspective of vehicular network engineers. In Proceedings of the sixteenth annual international conference on Mobile computing and networking(pp. 329-340). ACM.

Bang, S., \& Ahn, S. (2017). Platooning Strategy for Connected and Autonomous Vehicles: Transition from Light Traffic. Transportation Research Record: Journal of the Transportation Research Board, (2623), 73-81.

Bose, A., \& Ioannou, P. (1999). Analysis of traffic flow with mixed manual and semi-automated vehicles. In American Control Conference, 1999. Proceedings of the 1999 (Vol. 3, pp. 21732177). IEEE.

Carlson RC, Papamichail I, Papageorgiou M, \&Messmer A (2010). Optimal motorway traffic flow control involving variable speed limits and ramp metering. Transportation Science; 44, 238253.

Chang GL, Park SY, \& Paracha J (2011). Intelligent transportation system field demonstration: integration of variable speed limit control and travel time estimation for a recurrently congested highway. Transportation Research Record; 2243, 55-66.

Chen, D., S. Ahn, M. Chitturi, and \& D. A. Noyce (2017). Towards Vehicle Automation: Roadway Capacity Formulation for Traffic Mixed with Regular and Automated Vehicles. Transportation Research Part B: Methodological, Vol. 100, pp. 196-221. https://doi.org/10.1016/j.trb.2017.01.017. 
Cook, P. A. (2007). Stable control of vehicle convoys for safety and comfort. IEEE transactions on automatic control, 52(3), 526-531.

Dey, Kakan Chandra, et al (2016). "Vehicle-to-vehicle (V2V) and vehicle-to-infrastructure (V2I) communication in a heterogeneous wireless network-Performance evaluation." Transportation Research Part C: Emerging Technologies 68: 168-184.

Dresner, K., \& Stone, P. (2008). A multiagent approach to autonomous intersection management. Journal of artificial intelligence research, 31, 591-656.

Flint, M., Polycarpou, M., \& Fernandez-Gaucherand, E. (2002). Cooperative path-planning for autonomous vehicles using dynamic programming. IFAC Proceedings Volumes, 35(1), 481-486.

Ghiasi, A., Hussain, O., Qian, Z. S., \& Li, X. (2017). A mixed traffic capacity analysis and lane management model for connected automated vehicles: A Markov chain method. Transportation Research Part B: Methodological, 106, 266-292.

Gong, S., Shen, J., \& Du, L. (2016). Constrained optimization and distributed computation based car following control of a connected and autonomous vehicle platoon. Transportation Research Part B: Methodological, 94, 314-334.

Hadiuzzaman M, \& Qiu TZ (2012). Cell transmission model-based variable speed limit control for freeways. TRB 91st Annual Meeting.

Hafeez, K. A., Zhao, L., Ma, B., \& Mark, J. W. (2013). Performance analysis and enhancement of the DSRC for VANET's safety applications. IEEE Transactions on Vehicular Technology, 62(7), 3069-3083.

Hao, H., \& Barooah, P. (2013). Stability and robustness of large platoons of vehicles with double-integrator models and nearest neighbor interaction. International Journal of Robust and Nonlinear Control, 23(18), 2097-2122.

He, X., Liu, H. X., \& Liu, X. (2015). Optimal vehicle speed trajectory on a signalized arterial with consideration of queue. Transportation Research Part C: Emerging Technologies, 61, 106120.

Hegyi A, De Schutter B, \& Hellendoorn J (2006). Optimal coordination of variable speed limits to suppress shock waves, IEEE Transactions on Intelligent Transportation Systems; 6, 102-112.

Horowitz, R., \& Varaiya, P. (2000). Control design of an automated highway system. Proceedings of the IEEE, 88(7), 913-925.

Huang, K., Yang, X., Mi, C., \& Lu, Y., (2018) “Ecological Driving System for Connected/Automated Vehicle using a Two-Stage Control Hierarchy”, IEEE Transactions on Intelligent Transportation Systems, accepted with minor revision.

Jiang, D., Taliwal, V., Meier, A., Holfelder, W., \& Herrtwich, R. (2006). Design of 5.9 GHz DSRC-based vehicular safety communication. IEEE Wireless Communications, 13(5). 
Kamalanathsharma, R. K., \& Rakha, H. A. (2013). Multi-stage dynamic programming algorithm for eco-speed control at traffic signalized intersections. In Intelligent Transportation Systems(ITSC), 2013 16th International IEEE Conference on (pp. 2094-2099). IEEE.

Kang KP (2006). Development of optimal control strategies for freeway work zone operations. Ph. D. dissertation, Department of Civil \& Environmental Engineering, University of Maryland, 2006.

Kenney, J. B. (2011). Dedicated short-range communications (DSRC) standards in the United States. Proceedings of the IEEE, 99(7), 1162-1182.

Kwon E, Brannan D, Shouman K, Isackson C, \& Arseneau B (2007). Development and field evaluation of variable advisory speed limit system for work zones. Transportation Research Record; 2015, 12-18.

Lee, J., \& Park, B. (2012). Development and evaluation of a cooperative vehicle intersection control algorithm under the connected vehicles environment. IEEE Transactions on Intelligent Transportation Systems, 13(1), 81-90.

Lin PW, Kang KP, \&Chang GL (2004). Exploring the effectiveness of variable speed limit controls on highway work-zone operations. Journal of Intelligent Transportation System; 8(3), 155-168.

Lin, F., Fardad, M., \& Jovanovic, M. R. (2012). Optimal control of vehicular formations with nearest neighbor interactions. IEEE Transactions on Automatic Control, 57(9), 2203-2218.

Lioris, J., Pedarsani, R., Tascikaraoglu, F. Y., \& Varaiya, P. (2017). Platoons of connected vehicles can double throughput in urban roads. Transportation Research Part C: Emerging Technologies, 77, 292-305.

Lu, C. Y. (2016). Integrated Variable Speed Limit and Ramp Metering Control for Managing Recurrent Freeway Congestion. Ph.D Dissertation. University of Maryland.

Lu, N., Cheng, N., Zhang, N., Shen, X., \& Mark, J. W. (2014). Connected vehicles: Solutions and challenges. IEEE internet of things journal, 1(4), 289-299.

Lyles R, Taylor WC, \& Grossklaus J (2003). Field test of variable speed limits in work zones (in Michigan). Michigan Department of Transportation.

Lyu, P., Lin Y., Wang, L., \& Yang, X., (2017), Variable Speed Limit Control for Delay and Crash Reductions at Freeway Work Zone Area, Journal of Transportation Engineering, Part A: Systems, vol. 143 (12): 04017062.

Ma, J., Li, X., Zhou, F., Hu, J., \& Park, B. B. (2017). Parsimonious shooting heuristic for trajectory design of connected automated traffic part II: Computational issues and optimization. Transportation Research Part B: Methodological, 95, 421-441. 
Ma, X., Chen, X., \& Refai, H. H. (2009). Performance and reliability of DSRC vehicular safety communication: a formal analysis. EURASIP Journal on Wireless Communications and Networking, 2009(1), 969164.

McNaughton, M. (2011). Parallel algorithms for real-time motion planning. Carnegie Mellon University.

Monteil, J., Billot, R., Sau, J., \& El Faouzi, N. E. (2014). Linear and weakly nonlinear stability analyses of cooperative car-following models. IEEE Transactions on Intelligent Transportation Systems, 15(5), 2001-2013.

Oh, H., Yae, C., Ahn, D., \& Cho, H. (1999). 5.8 GHz DSRC packet communication system for ITS services. In Vehicular Technology Conference, 1999. VTC 1999-Fall. IEEE VTS 50th(Vol. 4, pp. 2223-2227). IEEE.

Öncü, S., Ploeg, J., van de Wouw, N., \& Nijmeijer, H. (2014). Cooperative adaptive cruise control: Network-aware analysis of string stability. IEEE Transactions on Intelligent Transportation Systems, 15(4), 1527-1537.

Papageorgiou M, Kosmatopoulos E, \& Papamichail I (2008). Effects of variable speed limits on motorway traffic flow. Transportation Research Record 2008; 2047, 37-48.

Silva, C. M., Masini, B. M., Ferrari, G., \& Thibault, I. (2017). A survey on infrastructure-based vehicular networks. Mobile Information Systems, 2017.

Sisiopiku VP, Sullivan AJ, \& Fadel G (2009). Implementing active traffic management strategies in the US. University of Transportation Center for Alabama, 2009.

Smulders S (1990). Control of freeway traffic flow by variable speed signs. Transportation Research Part B; 24, 111-132.

Taliwal, V., Jiang, D., Mangold, H., Chen, C., \& Sengupta, R. (2004). Empirical determination of channel characteristics for DSRC vehicle-to-vehicle communication. In Proceedings of the 1st ACM international workshop on Vehicular ad hoc networks (pp. 88-88). ACM.

Wang, M., Daamen, W., Hoogendoorn, S. P., \& van Arem, B. (2014). Rolling horizon control framework for driver assistance systems. Part I: Mathematical formulation and non-cooperative systems. Transportation research part C: emerging technologies, 40, 271-289.

Wang, Z., \& Hassan, M. (2008). How much of DSRC is available for non-safety use?. In Proceedings of the fifth ACM international workshop on VehiculAr Inter-NETworking (pp. 23-29). ACM.

Wei, Y., Avcı, C., Liu, J., Belezamo, B., Aydın, N., Li, P. T., \& Zhou, X. (2017). Dynamic programming-based multi-vehicle longitudinal trajectory optimization with simplified car following models. Transportation Research Part B: Methodological, 106, 102-129. 
Wu, X., He, X., Yu, G., Harmandayan, A., \& Wang, Y. (2015). Energy-optimal speed control for electric vehicles on signalized arterials. IEEE Transactions on Intelligent Transportation Systems, 16(5), 2786-2796.

Xu, Q., Mak, T., Ko, J., \& Sengupta, R. (2004). Vehicle-to-vehicle safety messaging in DSRC. In Proceedings of the 1st ACM international workshop on Vehicular ad hoc networks (pp. 1928). ACM.

Yang, X., Lu, Y. C., \& Chang, G. L. (2015). Exploratory analysis of an optimal variable speed control system for a recurrently congested freeway bottleneck. Journal of Advanced Transportation, 49(2), 195-209.

Yang, X., Lu, Y., \& Lin, Y. (2016). Optimal variable speed limit control system for freeway work zone operations. Journal of computing in civil engineering, Vol 31(1), 04016044.

Yin, J., ElBatt, T., Yeung, G., Ryu, B., Habermas, S., Krishnan, H., \& Talty, T. (2004). Performance evaluation of safety applications over DSRC vehicular ad hoc networks. In Proceedings of the 1st ACM international workshop on Vehicular ad hoc networks (pp. 1-9). ACM.

Zhou, F., Li, X., \& Ma, J. (2017). Parsimonious shooting heuristic for trajectory design of connected automated traffic part I: Theoretical analysis with generalized time geography. Transportation Research Part B: Methodological, 95, 394-420. 


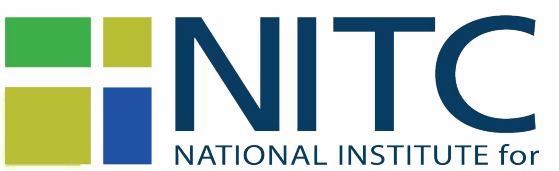

TRANSPORTATION and COMMUNITIES

Transportation Research and Education Center

Portland State University

1900 S.W. Fourth Ave., Suite 175

Portland, OR 97201 